\title{
A Nonconstant Shape Parameter-Dependent Competing Risks' Model in Accelerate Life Test Based on Adaptive Type-II Progressive Hybrid Censoring
}

\author{
Yan Wang $\mathbb{D}^{1,2}$ Yimin Shi, ${ }^{1}$ and $M i n \mathrm{Wu}^{3}$ \\ ${ }^{1}$ Scholl of Mathematics and Statistics, Northwestern Polytechnical University, Xi'an 710072, China \\ ${ }^{2}$ School of Science, Xi'an Polytechnic University, Xi'an 710048, China \\ ${ }^{3}$ School of Economics and Management, Shanghai Maritime University, Shanghai 201306, China
}

Correspondence should be addressed to Yan Wang; wywzyf@126.com

Received 31 December 2020; Revised 19 February 2021; Accepted 27 March 2021; Published 16 April 2021

Academic Editor: Emilio Gómez-Déniz

Copyright (c) 2021 Yan Wang et al. This is an open access article distributed under the Creative Commons Attribution License, which permits unrestricted use, distribution, and reproduction in any medium, provided the original work is properly cited.

In this paper, the dependent competing risks' model is considered in the constant-stress accelerated life test under the adaptive type-II progressive hybrid-censored scheme. The dependency between failure causes is modeled by Marshall-Olkin bivariate Gompertz distribution. The scale and shape parameters in the model both change with the stress levels, and the failure causes of some test units are unknown. Then, the maximum likelihood estimations and approximation confidence intervals of the unknown parameters are considered. And, the necessary and sufficient condition is established for the existence and uniqueness of the maximum likelihood estimations for unknown parameters. The Bayes approach is also employed to estimate the unknown parameters under suitable prior distributions. The Bayes estimations and highest posterior credible intervals of the unknown parameters are obtained. Finally, a simulation experiment has been performed to illustrate the methods proposed in this paper.

\section{Introduction}

The accelerated life test (ALT) has been proposed as an effective method to obtain failure information of the highly reliable units, which frequently appears in electronics, automation, or aerospace fields, and its test units are subjected to the higher-than-use stress levels. In the ALT, it is generally assumed that the scale parameters of life distribution change with the stress levels, while the other shape parameter is constant during the test. However, some research results have shown that the shape parameters change with the stress levels. Researchers such as $\mathrm{Li}$ et al. [1] discovered that the shape parameter of life distribution for electrolyte changes with the increase of voltage, when electrolyte is subjected to an electric test. Hiergeist et al. [2] discovered that the shape parameter of life distribution changes with the corresponding temperature in the capacitor test.

In the competing risks' model, there are more than one failure causes, and each cause possibly leads to the final failure of the unit. The unit operation may cause correlation among the failure causes, and failure causes may make the unit fail early. So, a failure cause is commonly associated with the other failure causes, and it is not usually possible to study the units with the isolated failure cause, and it is necessary to access each failure cause in the presence of other failure causes. To construct the dependence between failure causes, it is natural to consider a bivariate or multivariate distribution for the lifetimes. Among the multivariate distribution, the Marshal1-Olkin family distributions in [3] are important bivariate distributions, and they have a parameter that describes the correlation structure between marginal distributions. Thus, they are used to describe the dependence between the failure causes. For example, Guan et al. [4] discussed the Bayes estimations for Marshall-Olkin bivariate exponential (MOBE) distribution. Shen and $\mathrm{Xu}$ [5] analyzed the statistical inference of dependent competing risks' model using the Marshall-Olkin bivariate Weibull (MOBW) distribution. Bai et al. [6] considered the maximum likelihood estimations (MLEs) and the 
confidence intervals (CIs) for MOBW distribution under the type-I progressive interval censoring scheme. Kundu and Gupta [7] discussed the Bayes estimations (BEs) of the MOBW distribution. Feizjavadian and Hashemi [8] investigated the MOBW distribution under progressive hybrid censoring.

In the competing risks' model, the failure data of each unit is represented by a bivariate form data, which is composed of the failure time and corresponding failure cause. In the previous studies on competing risks' models, it is usually assumed that the failure cause of unite can be observed. However, in some cases, because of the high cost or the other reasons, the determination cause of failure may be difficult to obtain $[9,10]$. Thus, the failure time is observed, while no corresponding failure occurs.

Censoring has become a part of the life test where the test terminates before all of the undergoing units fail. This is done to save and reduce duration of the experiment and cost incurred in the test. Among the censoring schemes, the adaptive type-II progressive hybrid censored scheme (AT-II PHCS), introduced by $\mathrm{Ng}$ et al. [11], is one of the popular schemes, where one can obtain effective failure numbers for the statistical inference and also speed up the test when the test reaches the given time. The AT-II PHCS can be described as follows. Suppose $n$ identical units are placed on the life test. The observed failure number $m(m<n)$ is fixed in advance, and the experiment time is allowed to run over the prefixed time $\tau, \tau \in(0, \infty)$. The progressive censoring scheme $\left(R_{1}, R_{2}, \ldots, R_{m}\right)$ is allowed to change according to the $m$ failure times $T_{1: m: n}, T_{2: m: n}, \ldots, T_{m: m: n}$. For convenience, let $T_{i}=T_{i: m: n}, i=1,2, \ldots, m$. At the time of the first failure $T_{1}$, $R_{1}$ units are randomly removed from the remaining $n-1$ units. Similarly, at the second failure $T_{2}, R_{2}$ units from the remaining $n-2-R_{1}$ units are randomly removed and so on. If the $m$ th observed failure occurs before time $\tau$ (i.e., $T_{m}<\tau$ ), the test stops at time $T_{m}$, and all remaining $R_{m}$ units are removed, where $R_{m}=n-m-\sum_{i=1}^{m} R_{i}$. Otherwise, if $T_{J}<\tau<T_{J+1}$, where $J+1<m$, and we adapt the number of units removed from the test upon failures by setting $R_{I+1}=R_{J+2}=\ldots=R_{m-1}=0$ and $R_{m}=n-m-\sum_{i=1}^{J} R_{i}$. In this case, the actually applied progressive censored scheme is $\left(R_{1}, \ldots, R_{J}, 0, \ldots, 0, n-m-\right.$ $\left.\sum_{i=1}^{J} R_{i}\right)$. Presently, some scholars discussed the AT-II PHCS, i.e., Cramer and Iliopoulos [12] derived some distributional results and showed that maximum likelihood estimators coincide with those in deterministic progressive type-II censoring. Hemmati and Khorram [13] discussed the AT-II PHCS in the presence of the competing risk. Nassar and Abo-Kasem [14] adopted the frequents and Bayse methods to obtain the estimations of the unknown parameters for inverse Weibull distribution under AT-II PHCS.

Different from the previous studies, the dependent competing risks' model using Marshall-Olkin bivariate Gompertz (MOBG) with nonconstant shape parameter in constant stress accelerated life test (CSALT) is considered under AT-IIPHCS. In addition, we assume that the failure causes of some units are unknown. The Gompertz distribution is first introduced by Gompertz [15] to describe human mortality and establish actuarial tables. This distribution is a generalization of the exponential distribution and has many real life applications, especially in medical and actuarial studies. To quote a select few, Doblhammer [16], Perozek [17], and Willemse and Koppelaar [18] adopted this distribution to describe the adult lifespan by demographers and actuaries. Finch and Pike [19] and Ricklefs and Scheuerlein [20] used the Gompertz distribution to fit the mortality data of birds and mammals. In addition, this distribution which is used as a statistical model is fitted the tumor growth in survival analysis and reliability. Statistical inference for the Gompertz distribution has received many authors' attention, such as Wu and Shi [21] considered the BEs of the Gompertz distribution in the competing risks' model under progressively hybrid censoring. Liu et al. [22] studied the estimation for stress-strength reliability of the system with multiple types of components based on the Gompertz distribution. Mohie El-Din et al. [23] investigated the MLEs and BEs of the Gompertz distribution based on the generalized progressively hybrid censored scheme. Dey et al. [24] considered the properties and the estimation of unknown parameters of Gompertz distribution and so on.

This paper aims to investigate statistical analysis of the dependent competing risk model by using the MOBG distribution under CSALT with AT-II PHCS, where the shape and scale parameters are both changed with the stress levels. The rest of this paper is organized as follows. In Section 2, we describe the MOBG distribution and the accelerated dependent competing risks' model in which the shape and scale parameters are both changed with the stress levels and the failure causes of some units are unknown. In Section 3, MLEs and BEs of the unknown parameters are obtained based on the discrete distribution and gamma distribution. The CIs of the unknown parameters based on MLEs and BEs are considered in Section 4. The simulation results and real data analysis are showed in Section 5. Conclusions are provided in Section 6.

\section{Model Description}

2.1. The Marshall-Olkin Gompertz Distribution. The probability density function (PDF) and the cumulative distribution function (CDF) of the Gompertz distribution with parameters $\lambda$ and $\alpha$ can be represented as follows, respectively:

$$
\begin{aligned}
& f(t)=\alpha \exp (\lambda t) \exp \left\{-\frac{\alpha}{\lambda}(\exp (\lambda t)-1)\right\}, \quad t>0, \alpha>0, \lambda>0, \\
& F(t)=1-\exp \left\{-\frac{\alpha}{\lambda}(\exp (\lambda t)-1)\right\}, \quad t>0, \alpha>0, \lambda>0 .
\end{aligned}
$$

The reliability function of Gompertz distribution can be expressed as follows:

$$
R(t)=\exp \left\{-\frac{\alpha}{\lambda}(\exp (\lambda t)-1)\right\}, \quad t>0, \alpha>0, \lambda>0,
$$

where $\lambda$ is the shape parameter and $\alpha$ is the scale parameter. We denote the Gompertz distribution with parameters $\lambda$ and $\alpha$ as $\operatorname{GP}(\lambda, \alpha)$.

Suppose that the random variable $U_{i}, i=0,1$, and 2 , are independent of each other and $U_{i} \sim \operatorname{GP}\left(\lambda, \alpha_{i}\right)$. Let $X=\min \left(U_{0}, U_{1}\right)$ and $Y=\min \left(U_{0}, U_{2}\right)$, then the bivariate 
random variable $(X, Y)$ is followed by the MOBG distribution with shape parameter $\lambda$ and scale parameters $\alpha_{0}, \alpha_{1}$, and $\alpha_{2}$, and it is denoted by $\operatorname{MOBG}\left(\lambda, \alpha_{0}, \alpha_{1}, \alpha_{2}\right)$. Thus, the joint survival function of $(X, Y)$ is shown as follows:

$$
\begin{aligned}
S_{X, Y}(x, y) & =P(X>x, Y>y), \\
& =P\left(U_{0}>\max (x, y), U_{1}>x, U_{2}>y\right), \\
& =S\left(\max (x, y) \mid \lambda, \alpha_{0}\right) S\left(x \mid \lambda, \alpha_{1}\right) S\left(y \mid \lambda, \alpha_{2}\right), \\
& = \begin{cases}S\left(x \mid \lambda, \alpha_{1}\right) S\left(y \mid \lambda, \alpha_{0}+\alpha_{2}\right), & \text { if } x<y, \\
S\left(x \mid \lambda, \alpha_{0}+\alpha_{1}\right) S\left(y \mid \lambda, \alpha_{2}\right), & \text { if } x>y, \\
S\left(z \mid \lambda, \alpha_{0}+\alpha_{1}+\alpha_{2}\right), & \text { if } x=y=z .\end{cases}
\end{aligned}
$$

And, the joint PDF of $(X, Y)$ can be obtained as the following expression:

$$
f_{X, Y}(x, y)= \begin{cases}f_{1}(x, y), & \text { if } x<y, \\ f_{2}(x, y), & \text { if } x<y, \\ f_{0}(x, y), & \text { if } x=y=z,\end{cases}
$$

where

$$
\begin{aligned}
f_{1}(x, y) & =f\left(x \mid \lambda, \alpha_{1}\right) f\left(y \mid \lambda, \alpha_{0}+\alpha_{2}\right), \\
f_{2}(x, y) & =f\left(x \mid \lambda, \alpha_{2}\right) f\left(y \mid \lambda, \alpha_{0}+\alpha_{1}\right), \\
f_{0}(z) & =\left(\frac{\alpha_{0}}{\left(\alpha_{0}+\alpha_{1}+\alpha_{2}\right)}\right) f\left(z \mid \lambda, \alpha_{0}+\alpha_{1}+\alpha_{2}\right) .
\end{aligned}
$$

2.2. Model Description. In this section, the dependent competing risks' model in CSALT under AT-II PHCS is considered.

Let $S_{1}<\ldots<S_{l}$ be the accelerated stress levels and $S_{0}$ be the normal stress level. The AT-II PHCS under CSALT can be run as follows. Under the $i$ th stress level $S_{i}, i=1,2 \ldots, l$, and suppose $n_{i}$ identical units are put on the life test, the observed failure number $m_{i}\left(m_{i}<n_{i}\right)$, terminal time $\tau$, and progressive censoring scheme $\left(R_{i 1}, R_{i 2}, \ldots, R_{i m_{i}}\right)$ are given in advance. For the given $\tau$, if $T_{i J_{i}}<\tau<T_{i J_{i}+1}$, where $J_{i}+1<m_{i}$, the removed units from the test upon failures is $R_{i j}^{*}=R_{i j} I\left(T_{i J_{i}}<\tau\right), j=1,2, \ldots, m_{i}-1$, and $R_{i m_{i}}^{*}=\sum_{j=J_{i}+1}^{m_{i}}$ $R_{i j}$. Otherwise, $R_{i j}^{*}=R_{i j}, j=1,2, \ldots, m_{i}$.

The following basic assumptions are made for the statistical inference of the dependent competing risks' model.

A1: under the stress level $S_{i}, i=1,2, \ldots, l$; for the $j$ th unit, there are two random variables $X_{i j}$ and $Y_{i j}$, which are the failure time under first and second failure causes, respectively. Thus, the failure time of the unit is $T_{i j}=\min \left(X_{i j}, Y_{i j}\right)$.

A2: under the stress level $S_{i}, i=1,2, \ldots, l$; the pair of random variable $\left(X_{i j}, Y_{i j}\right)$ follows a $\operatorname{MOBG}\left(\lambda_{i}, \alpha_{i 0}\right.$, $\left.\alpha_{i 1}, \alpha_{i 2}\right) . X_{i j}$ follows $\operatorname{GP}\left(\lambda_{i}, \alpha_{i 01}\right), Y_{i j}$ follows $\operatorname{GP}\left(\lambda_{i}\right.$, $\left.\alpha_{i 02}\right)$, and $T_{i j}=\min \left(X_{i j}, Y_{i j}\right)$ follows $\operatorname{GP}\left(\lambda_{i}, \alpha_{i}\right)$ where, $\alpha_{i 01}=\alpha_{i 0}+\alpha_{i 1}, \alpha_{i 02}=\alpha_{i 0}+\alpha_{i 2}$, and $a_{i}=a_{i 0}+a_{i 1}+a_{i 2}$. A3: the accelerated function of the scale parameter is log-linear:

$$
\log \alpha_{i k}=a_{i k}+b_{i k} \varphi\left(S_{i}\right), \quad i=1,2, \ldots, l, k=0,1 \text {, and } 2 .
$$

The accelerated function of the shape parameter is loglinear:

$$
\log \lambda_{i}=a+b \varphi\left(S_{i}\right), \quad i=1,2, \ldots, l,
$$

where $a, b, a_{i k}$, and $b_{i k}$ are the accelerated factors and the unknown parameters and $\varphi\left(S_{i}\right)$ is the known function of the stress level $S_{i}$

Based on the model description, we can obtain the adaptive type-II progressive hybrid censored competing risks' data $\left(T_{i 1}, c_{i 1}, R_{i 1}^{*}\right),\left(T_{i 2}, c_{i 2}, R_{i 2}^{*}\right), \ldots,\left(T_{i m_{i}}, c_{i m_{i}}, R_{i m_{i}}^{*}\right)$, where $c_{i j} \in\{0,1,2, *\}$ is failure cause of the $i$ th unit, and it is defined as follows:

$$
c_{i j}= \begin{cases}0, & \text { if } X_{i j}=Y_{i j}, \\ 1, & \text { if } X_{i j}<Y_{i j}, \quad j=1,2, \ldots, m_{i}, \\ 2, & \text { if } X_{i j}>Y_{i j},\end{cases}
$$

while $c_{i j}=*$ indicates that the failure cause is uncertain.

Under the stress level $S_{i}, i=1,2, \ldots, l$, let $m_{i k}=\sum_{j=1}^{m_{i}}$ $\delta_{k}\left(c_{i j}\right), k=0,1,2$ represent the failure numbers of those caused by both risks, first risk and second risk, where $\delta_{k}(\cdot), k=0,1$, and 2 , is the indicator function; $\delta_{k}\left(c_{i j}\right)=1$, if $c_{i j}=k ; \delta_{k}\left(c_{i j}\right)=0$, if $c_{i j} \neq k$. In addition, $m_{i 3}=\sum_{j=1}^{m} \delta$ $\left(c_{i j}=*\right)$ represents the numbers of the failure units which have the failure time, while not having the corresponding failure cause. Let $m_{i 0}+m_{i 1}+m_{i 2}=m_{i}^{*}$ and $m_{i}^{*}+m_{i 3}=m_{i}$, and we can obtain the following result.

Theorem 1. Under the stress level $S_{i}, i=1,2, \ldots, l$,

$$
\left(m_{i 0}, m_{i 1}, m_{i 2}\right) \sim \text { multinomial }\left(m_{i}^{*}, \frac{\alpha_{i 0}}{\alpha_{i}}, \frac{\alpha_{i 1}}{\alpha_{i}}, \frac{\alpha_{i 2}}{\alpha_{i}}\right),
$$

where $\alpha_{i}=\alpha_{i 0}+\alpha_{i 1}+\alpha_{i 2}$.

Proof. Under the stress level $S_{i}, i=1,2, \ldots, l$, each unit failure is caused by one of $X_{i j}>Y_{i j}, X_{i j}<Y_{i j}$, or $X_{i j}=Y_{i j}$. Probability of each case is

$$
\begin{aligned}
& P\left(X_{i j}<Y_{i j}\right)=\int_{0}^{+\infty} \int_{0}^{y_{i j}} f_{1}\left(x_{i j}, y_{i j}\right) \mathrm{d} x_{i j} \mathrm{~d} y_{i j}=\frac{\alpha_{i 1}}{\alpha_{i}}, \\
& P\left(Y_{i j}<X_{i j}\right)=\int_{0}^{+\infty} \int_{0}^{x_{i j k}} f_{1}\left(x_{i j}, y_{i j}\right) \mathrm{d} y_{i j} \mathrm{~d} x_{i j}=\frac{\alpha_{i 2}}{\alpha_{i}} .
\end{aligned}
$$

For the given $m_{i}^{*}, m_{i 0}, m_{i 1}$, and $m_{i 2}$, we can obtain $P\left(Y_{i j}=X_{i j}\right)=\alpha_{i 0} / \alpha_{i}$ by

$$
\begin{aligned}
& \int_{0}^{+\infty} \int_{0}^{y_{i j}} f_{1}\left(x_{i j}, y_{i j}\right) \mathrm{d} x_{i j} \mathrm{~d} y_{i j} \\
& \quad+\int_{0}^{+\infty} \int_{0}^{x_{i j}} f_{2}\left(x_{i j}, y_{i j}\right) \mathrm{d} y_{i j} \mathrm{~d} x_{i j}+\int_{0}^{+\infty} f_{0}(x) \mathrm{d} x=1 .
\end{aligned}
$$

Then, the theorem is completed. 


\section{Point Estimations}

3.1. Maximum Likelihood Estimations. Let $\mathbf{T}=\left(T_{i j}, i=1,2\right.$, $\left.\ldots, l, j=1,2, \ldots, m_{i}\right)$ is the censored sample in CSALT and $\mathbf{t}=\left(t_{i j}, i=1,2, \ldots, l, j=1,2, \ldots, m_{i}\right) \quad$ is the sample observation. Let $\theta=\left(\lambda_{1}, \ldots, \lambda_{l}, a_{10}, a_{11}, a_{12}, \ldots, a_{l 0}, a_{l 1}, a_{l 2}\right)$; under the stress level $S_{i}, i=1,2, \ldots, l$, based on the dependent competing risks' data, the likelihood function can be expressed as follows:

$$
\begin{aligned}
l_{i}\left(t \mid \lambda_{i}, \alpha_{i 0}, \alpha_{i 1}, \alpha_{i 2}\right) \propto & \left.\prod_{j=1}^{m_{i}}\left(f_{0}\left(t_{i j}, t_{i j}\right)\right)^{\delta_{0}\left(c_{i j}\right)\left[\frac{-S_{X, Y}(x, y)}{\partial x} \mid\left(t_{i j}, t_{i j}\right)\right]^{\delta_{1}\left(c_{i j}\right)}\left[\frac{-S_{X, Y}(x, y)}{\partial y}\left(t_{i j}, t_{i j}\right)\right.} \mid\right]^{\delta_{2}\left(c_{i j}\right)} \\
& \cdot\left(f_{0}\left(t_{i j}, t_{i j}\right)+\left.\frac{-S_{X, Y}(x, y)}{\partial x}\right|_{\left(t_{i j}, t_{i j}\right)}+\left.\frac{-S_{X, Y}(x, y)}{\partial y}\right|_{\left(t_{i j}, t_{i j}\right)}\right)^{\delta\left(c_{i j}=*\right)}\left(S_{X, Y}(x, y) \mid\right. \\
\left(t_{i j}, t_{i j}\right) & )^{R_{i j}^{*}},
\end{aligned}
$$

where

$$
\begin{aligned}
f_{0}\left(t_{i j}, t_{i j}\right) & =\alpha_{i 0} \exp \left(\lambda_{i} t_{i j}\right) \exp \left\{-\frac{\left(\alpha_{i 0}+\alpha_{i 1}+\alpha_{i 2}\right)}{\lambda_{i}}\left(\exp \left(\lambda_{i} t_{i j}\right)-1\right)\right\}, \\
\left.\frac{-S_{X, Y}(x, y)}{\partial x}\right|_{\left(t_{i j}, t_{i j}\right)} & =\alpha_{i 1} \exp \left(\lambda_{i} t_{i j}\right) \exp \left\{-\frac{\left(\alpha_{i 0}+\alpha_{i 1}+\alpha_{i 2}\right)}{\lambda_{i}}\left(\exp \left(\lambda_{i} t_{i j}\right)-1\right)\right\}, \\
\left.\frac{-S_{X, Y}(x, y)}{\partial y}\right|_{\left(t_{i j}, t_{i j}\right)} & =\alpha_{i 2} \exp \left(\lambda_{i} t_{i j}\right) \exp \left\{-\frac{\left(\alpha_{i 0}+\alpha_{i 1}+\alpha_{i 2}\right)}{\lambda_{i}}\left(\exp \left(\lambda t_{i j}\right)-1\right)\right\}, \\
-\left.S_{X, Y}(x, y)\right|_{\left(t_{i j}, t_{i j}\right)} & =\exp \left\{-\frac{\left(\alpha_{i 0}+\alpha_{i 1}+\alpha_{i 2}\right)}{\lambda_{i}}\left(\exp \left(\lambda_{i} t_{i j}\right)-1\right)\right\} .
\end{aligned}
$$

So, we can obtain

$$
\begin{gathered}
l_{i}\left(t \mid \lambda, \alpha_{i 0}, \alpha_{i 1}, \alpha_{i 2}\right) \propto \alpha_{i 0}^{m_{i 0}} \alpha_{i 1}^{m_{i 1}} \alpha_{i 2}^{m_{i 2}} \alpha_{i}^{m_{i}-m_{i}^{*}} \\
\cdot \exp \left\{\lambda_{i} \sum_{j=1}^{m_{i}} t_{i j}-\lambda_{i}^{-1} \alpha_{i} A_{i}\right\}
\end{gathered}
$$

where $A_{i}=\sum_{j=1}^{m_{i}}\left(R_{i j}^{*}+1\right)\left(\exp \left(\lambda_{i} t_{i j}\right)-1\right)$.
The full likelihood function is

$$
L=\prod_{i=1}^{l} l_{i} .
$$

Based on equation (16), the log-likelihood function can be transformed into the following result:

$$
\ln L=\sum_{i=1}^{l}\left\{\left(\sum_{k=0}^{2} m_{i k} \ln \alpha_{i k}\right)+\left(m_{i}-m_{i}^{*}\right) \ln \alpha_{i}+\lambda_{i} \sum_{j=1}^{m_{i}} t_{i j}-\lambda_{i}^{-1} \alpha_{i} A_{i}\right\} .
$$

The MLEs of unknown parameters can be obtained by the maximum function in equation (17). By setting the first partial derivative of $\ln L$ with respect to $\lambda_{i}$ and $\alpha_{i k}, k=0,1$, and 2 , to zero, we can obtain following results:

$$
\begin{gathered}
\frac{\partial \ln L}{\partial \alpha_{i k}}=\frac{m_{i k}}{\alpha_{i k}}+\frac{m_{i}-m_{i}^{*}}{\alpha_{i 0}+\alpha_{i 1}+\alpha_{i 2}}-\frac{1}{\lambda_{i}} A_{i}=0, \quad k=0,1, \text { and } 2, \\
\frac{\partial \ln L}{\partial \lambda_{i}}=\sum_{j=1}^{m_{i}} t_{i j}+\lambda_{i}^{-2} \alpha_{i} A_{i}-\lambda_{i}^{-1} \alpha_{i} \sum_{j=1}^{m_{i}} t_{i j}\left(R_{i j}^{*}+1\right) \exp \left(\lambda_{i} t_{i j}\right)=0 .
\end{gathered}
$$


By solving equation (18), we can get the MLE of $\alpha_{i k}, k=0,1$, and 2 , as the following form:

$$
\widehat{\alpha}_{i k}=\frac{m_{i k} m_{i} \lambda_{i}}{m_{i}^{*} A_{i}}, \quad k=0,1, \text { and } 2 .
$$

Substituting $\widehat{\alpha}_{i k}, k=0,1$, and 2, into equation (19), we can obtain the equation for $\lambda_{i}$ :

$$
H\left(\lambda_{i}\right)=\sum_{j=1}^{m_{i}} t_{i j}+\lambda_{i}^{-1} m_{i}-\frac{m_{i} \sum_{j=1}^{m_{i}} t_{i j}\left(R_{i j}^{*}+1\right) \exp \left(\lambda_{i} t_{i j}\right)}{A_{i}}=0 .
$$

Theorem 2. Based on the censored data $t_{i 1} \leq t_{i 2} \leq \ldots \leq t_{i j} \leq \ldots$ $\leq t_{\text {im }_{i}}$ and the progressive scheme $R_{i j}^{*}, j=1,2, \ldots, m_{i}$, the MLEs $\widehat{\lambda}_{i}$ and $\widehat{\alpha}_{i k}, k=0,1$, and 2, of the parameters $\lambda_{i}$ and $\alpha_{i k}, k=0,1$, and 2 , exist and are unique if and only if

$$
2 \sum_{j=1}^{m_{i}} t_{i j} \sum_{j=1}^{m_{i}}\left(R_{i j}^{*}+1\right) t_{i j}>m_{i} \sum_{j=1}^{m_{i}}\left(R_{i j}^{*}+1\right) t_{i j}^{2} \text {. }
$$

Proof. From equations (20) and (21), we can obtain that $\hat{\lambda}_{i}$ and $\widehat{\alpha}_{i k}, k=0,1$, and 2 , exist and are unique by showing that $\hat{\lambda}_{i}$ exists and is unique if and only if condition (22) holds. The derivative of $H\left(\lambda_{i}\right)$ can be expressed as follows:

$$
H^{\prime}\left(\lambda_{i}\right)=-\frac{m_{i}}{\lambda_{i}^{2}}-\left[\frac{m_{i} \sum_{j=1}^{m_{i}}\left(R_{i j}^{*}+1\right) \exp \left(\lambda_{i} t_{i j}\right) t_{i j}^{2}}{\sum_{j=1}^{m_{i}}\left(R_{i j}^{*}+1\right)\left(\exp \left(\lambda_{i} t_{i j}\right)-1\right)}-\frac{m_{i}\left(\sum_{j=1}^{m_{i}}\left(R_{i j}^{*}+1\right) \exp \left(\lambda_{i} t_{i j}\right) t_{i j}\right)^{2}}{\left(\sum_{j=1}^{m_{i}}\left(R_{i j}^{*}+1\right)\left(\exp \left(\lambda_{i} t_{i j}\right)-1\right)\right)^{2}}\right] .
$$

Let $z_{j}=z_{j}\left(\lambda_{i}\right)=\lambda_{i} t_{i j}$, we have

$$
\begin{aligned}
H^{\prime}\left(\lambda_{i}\right) & =-\frac{m_{i}}{\lambda_{i}^{2}}\left[1+\frac{\sum_{j=1}^{m_{i}}\left(R_{i j}^{*}+1\right) \exp \left(z_{j}\right) z_{j}^{2}}{\sum_{j=1}^{m_{i}}\left(R_{i j}^{*}+1\right)\left(\exp \left(z_{j}\right)-1\right)}-\frac{\left(\sum_{j=1}^{m_{i}}\left(R_{i j}^{*}+1\right) \exp \left(z_{j}\right) z_{j}\right)^{2}}{\left(\sum_{j=1}^{m_{i}}\left(R_{i j}^{*}+1\right)\left(\exp \left(z_{j}\right)-1\right)\right)^{2}}\right], \\
& =-\frac{m_{i}}{\lambda_{i}^{2}}\left[\frac{H_{1}\left(\lambda_{i}\right)}{\sum_{j=1}^{m_{i}}\left(R_{i j}^{*}+1\right)\left(\exp \left(z_{j}\right)-1\right)}+\frac{H_{2}\left(\lambda_{i}\right)}{\left(\sum_{j=1}^{m_{i}}\left(R_{i j}^{*}+1\right)\left(\exp \left(z_{j}\right)-1\right)\right)^{2}}\right],
\end{aligned}
$$

where

$$
\begin{aligned}
& H_{1}\left(\lambda_{i}\right)=\sum_{j=1}^{m_{i}}\left(R_{i j}^{*}+1\right) \frac{1}{\left(\exp \left(z_{j}\right)-1\right)}\left[\left(\exp \left(z_{j}\right)-1\right)^{2}-z_{i}^{2} \exp \left(z_{j}\right)\right], \\
& H_{2}\left(\lambda_{i}\right)=\sum_{j=1}^{m_{i}}\left(R_{i j}^{*}+1\right)\left(\exp \left(z_{j}\right)-1\right) \sum_{j=1}^{m_{i}}\left(R_{i j}^{*}+1\right) \frac{\left(z_{i} \exp \left(z_{j}\right)\right)^{2}}{\left(\exp \left(z_{j}\right)-1\right)}-\left(\sum_{j=1}^{m_{i}}\left(R_{i j}^{*}+1\right) \exp \left(z_{j}\right) z_{j}\right)^{2} .
\end{aligned}
$$

Since $\left(\exp \left(z_{j}\right)-1\right)^{2}>z_{i}^{2} \exp \left(z_{j}\right)$ for all $z_{j}>0$, we have $H_{1}\left(\lambda_{i}\right)>0$.

Now, let

$$
\begin{aligned}
& h_{j}=\left[\left(R_{i j}^{*}+1\right)\left(\exp \left(z_{j}\right)-1\right)\right]^{1 / 2}, \\
& g_{j}=\left[\frac{\left(R_{i j}^{*}+1\right)}{\left(\exp \left(z_{j}\right)-1\right)}\right]^{1 / 2} z_{i} \exp \left(z_{j}\right) .
\end{aligned}
$$

Then, we have

$$
H_{2}\left(\lambda_{i}\right)=\sum_{j=1}^{m_{i}} h_{j}^{2} \sum_{j=1}^{m_{i}} g_{j}^{2}-\left(\sum_{j=1}^{m_{i}} h_{j} g_{j}\right)^{2} .
$$

By using the Cauchy-Schwarz inequality, we can obtain $H_{2}\left(\lambda_{i}\right) \geq 0$. Then, it follows that $H^{\prime}\left(\lambda_{i}\right)<0$ which implies that $H\left(\lambda_{i}\right)$ is a decrease function. Therefore, equation (21) has the unique root $\widehat{\lambda}_{i} \in(0, \infty)$ if and only if $H(0)>0$ and $H(\infty)<0$ : 


$$
\begin{aligned}
H(0) & =\sum_{j=1}^{m_{i}} t_{i j}+m_{i} \lim _{\lambda_{i} \longrightarrow 0}\left[\frac{1}{\lambda_{i}}-\frac{\sum_{j=1}^{m_{i}} t_{i j}\left(R_{i j}^{*}+1\right) \exp \left(\lambda_{i} t_{i j}\right)}{\sum_{j=1}^{m_{i}}\left(R_{i j}^{*}+1\right)\left(\exp \left(\lambda_{i} t_{i j}\right)-1\right)}\right], \\
& =\sum_{j=1}^{m_{i}} t_{i j}-\frac{m_{i}}{2} \frac{\sum_{j=1}^{m_{i}}\left(R_{i j}^{*}+1\right) t_{i j}^{2}}{\sum_{j=1}^{m_{i}}\left(R_{i j}^{*}+1\right) t_{i j}}, \\
& =\frac{2 \sum_{j=1}^{m_{i}} t_{i j} \sum_{j=1}^{m_{i}}\left(R_{i j}^{*}+1\right) t_{i j}-m_{i} \sum_{j=1}^{m_{i}}\left(R_{i j}^{*}+1\right) t_{i j}^{2}}{2 \sum_{j=1}^{m_{i}}\left(R_{i j}^{*}+1\right) t_{i j}} .
\end{aligned}
$$

If and only if equation (22) is satisfied, $H(0)>0$ :

$$
\begin{aligned}
H(\infty) & =\sum_{j=1}^{m_{i}} t_{i j}-m_{i} \lim _{\lambda_{i} \longrightarrow \infty} \frac{\sum_{j=1}^{m_{i}} t_{i j}\left(R_{i j}^{*}+1\right) \exp \left(\lambda_{i} t_{i j}\right)}{\sum_{j=1}^{m_{i}}\left(R_{i j}^{*}+1\right)\left(\exp \left(\lambda_{i} t_{i j}\right)-1\right)}, \\
& =\sum_{j=1}^{m_{i}} t_{i j}-m_{i} \lim _{\lambda_{i} \longrightarrow \infty} \frac{\sum_{j=1}^{m_{i}} t_{i j}^{2}\left(R_{i j}^{*}+1\right) \exp \lambda_{i}\left(t_{i j}-t_{i m_{i}}\right)}{\sum_{j=1}^{m_{i}} t_{i j}\left(R_{i j}^{*}+1\right) \exp \lambda_{i}\left(t_{i j}-t_{i m_{i}}\right)}, \\
& =\sum_{j=1}^{m_{i}}\left(t_{i j}-t_{i m_{i}}\right)<0 .
\end{aligned}
$$

Then, the theorem is completed.

Theorem 3. Using the MLE of the parameter $\hat{\alpha}_{i k}, k=0,1$, and 2 , we can get the MLE $\widehat{\vartheta}=\widehat{\alpha}_{i 0}+\widehat{\alpha}_{i 1}+\widehat{\alpha}_{i 2}$ of the parameter $\vartheta=\alpha_{i 0}+\alpha_{i 1}+\alpha_{i 2}$ having the following properties:

(1) $\widehat{\vartheta}$ is the asymptotic unbiased estimator of $\vartheta$

(2) $\widehat{\vartheta}$ is a consistent estimator of $\vartheta$

(3) $\widehat{\vartheta}$ is a sufficient statistics of $\vartheta$

Proof. From A2, $T_{i j}$ follows the Gompertz distribution with the shape parameter $\lambda_{i}$ and scale parameter $\alpha_{i}$. By the variable transformed method, the random variable $Y_{i j}=\left(\exp \left(\lambda_{i} T_{i j}\right)-1\right) / \lambda_{i}$ follows the exponential distribution with mean $1 / \alpha_{i}$.

$$
\begin{aligned}
& \text { Let } \\
& \begin{aligned}
Z_{i 1} & =n_{i} Y_{i 1}, \\
Z_{i 2} & =\left(n_{i}-R_{i 1}^{*}-1\right)\left(Y_{i 2}-Y_{i 1}\right), \\
& \ldots \\
Z_{i m_{i}} & =\left(n_{i}-R_{i 1}^{*}-\ldots R_{i m_{i}}^{*}-m_{i}-1\right)\left(Y_{i m_{i}}-Y_{i, m_{i}-1}\right) .
\end{aligned}
\end{aligned}
$$

We can obtain that $Z_{i 1}, Z_{i 2}, \ldots, Z_{i m_{i}}$ are independent and identical distribution as the exponential distribution with mean $1 / \alpha_{i}$. According to the relationship between the exponential distribution and gamma distribution, we have

$$
Z_{i j} \sim E\left(\alpha_{i}\right)=\alpha_{i} e^{-\alpha_{i} z_{i j}}=\frac{\alpha_{i}^{1}}{\Gamma(1)}\left(z_{i j}\right)^{1-1} e^{-\alpha_{i} z_{i j}} \sim \operatorname{Gamma}\left(1, \alpha_{i}\right)
$$

Let $W_{i}=Z_{i 1}+Z_{i 2}+\cdots Z_{i m_{i}}$, then $W_{i} \sim \operatorname{Gamma}\left(m_{i}, \alpha_{i}\right)$ :

$$
W_{i}=Z_{i 1}+Z_{i 2}+\cdots Z_{i m_{i}}=\frac{\sum_{j=1}^{m_{i}}\left(R_{i j}^{*}+1\right)\left(\exp \left(\lambda_{i} t_{i j}\right)-1\right) / \lambda=A_{i}}{\lambda_{i}} .
$$

Then, $\lambda_{i} / A_{i}$ follows the inverse gamma distribution with the parameters $m_{i}$ and $\alpha_{i}$, and it is denoted by $\lambda_{i} / A_{i} \sim \mathrm{IGa}$ $\left(m_{i}, \alpha_{i}\right)$.

Using equation (20),

$$
E\left(\widehat{\alpha}_{i 0}+\widehat{\alpha}_{i 1}+\widehat{\alpha}_{i 2}\right)=E\left(\frac{m_{i} \lambda_{i}}{A_{i}}\right)=\frac{\left(m_{i}\left(\alpha_{i 0}+\alpha_{i 1}+\alpha_{i 2}\right)\right)}{\left(m_{i}-1\right)} .
$$

Then, we have

$$
\lim _{m_{i} \longrightarrow \infty} E\left(\widehat{\alpha}_{i 0}+\widehat{\alpha}_{i 1}+\widehat{\alpha}_{i 2}\right)=\alpha_{i 0}+\alpha_{i 1}+\alpha_{i 2} .
$$

The proof of statement 1 is completed.

Using equation (20), we can obtain

$$
\operatorname{Var}\left(\widehat{\alpha}_{i 0}+\widehat{\alpha}_{i 1}+\widehat{\alpha}_{i 2}\right)=E\left(\frac{m_{i} \lambda_{i}}{A_{i}}\right)=\frac{m_{i}^{2}\left(\alpha_{i 0}+\alpha_{i 1}+\alpha_{i 2}\right)^{2}}{\left(\left(m_{i}-1\right)\left(m_{i}-2\right)\right)} .
$$

Then,

$$
\begin{aligned}
\lim _{m_{i} \longrightarrow \infty} \operatorname{Var}\left(\widehat{\alpha}_{i 0}+\widehat{\alpha}_{i 1}+\widehat{\alpha}_{i 2}\right) & =\frac{\left(\alpha_{i 0}+\alpha_{i 1}+\alpha_{i 2}\right)^{2} \lim _{m_{i} \longrightarrow \infty} m_{i}^{2}}{\left(\left(m_{i}-1\right)^{2}\left(m_{i}-2\right)\right)} \\
& =0 .
\end{aligned}
$$

The proof of statement 2 is completed.

Finally, under the stress level $S_{i}, i=1,2, \ldots, l$, the likelihood function can be written as follows:

$$
l_{i} \propto \alpha_{i 0}^{m_{i 0}} \alpha_{i 1}^{m_{i 1}} \alpha_{i 2}^{m_{i 2}} \alpha_{i}^{m_{i}-m_{i}^{*}} \exp \left\{\lambda_{i} \sum_{j=1}^{m_{i}} t_{i j}-\lambda_{i}^{-1}\left(\alpha_{i 0}+\alpha_{i 1}+\alpha_{i 2}\right) A_{i}\right\} \text {. }
$$

Based on equation (20), we can obtain

$$
A_{i}=\frac{\lambda_{i} m_{i}}{\left(\widehat{\alpha}_{i 0}+\widehat{\alpha}_{i 1}+\widehat{\alpha}_{i 2}\right)} \text {. }
$$

Then, the likelihood function can be written as follows:

$$
\begin{aligned}
& l_{i} \propto \alpha_{i 0}^{m_{i 0}} \alpha_{i 1}^{m_{i 1}} \alpha_{i 2}^{m_{i 2}} \alpha_{i}^{m_{i}-m_{i}^{*}} \exp \left\{\lambda_{i} \sum_{j=1}^{m_{i}} t_{i j}-m_{i}\left(\alpha_{i 0}+\alpha_{i 1}+\alpha_{i 2}\right) /\left(\widehat{\alpha}_{i 0}+\widehat{\alpha}_{i 1}+\widehat{\alpha}_{i 2}\right)\right\} \\
& \propto G\left(t_{i 1}, \ldots, t_{i m_{i}}\right) H\left(\alpha_{i 0}, \alpha_{i 1}, \alpha_{i 2}, \widehat{\alpha}_{i 0}, \widehat{\alpha}_{i 1}, \widehat{\alpha}_{i 2}\right) .
\end{aligned}
$$


The likelihood function can be decomposed into two functions $G\left(t_{i 1}, \ldots, t_{i m_{\mathrm{i}}}\right)$ and $H\left(\alpha_{i 0}, \alpha_{i 1}, \alpha_{i 2}, \widehat{\alpha}_{i 0}, \widehat{\alpha}_{i 1}, \widehat{\alpha}_{i 2}\right)$, where $G$ is only dependent on the data and $H$ is dependent on the parameters. This completes the proof of statement 3 and the theorem.

3.2. The Bayes Estimations. We assume that the parameters $\lambda_{i}, \alpha_{i 0}, \alpha_{i 1}$, and $\alpha_{i 2}$ are independent of each other. According to $\mathrm{Wu}$ and Shi [21], for the parameter $\lambda_{i}$, we select a finite number of values on the interval $(0, \infty)$, which is denoted by $\lambda_{i 1}, \lambda_{i 2}, \ldots, \lambda_{i r}$. The PDF of the parameters $\lambda_{i}$ is $\pi\left(\lambda_{i}=\right.$ $\left.\lambda_{i r}\right)=\pi_{i r}$, where $\sum_{r=1}^{q} \pi_{i r}=1,0 \leq \pi_{i r} \leq 1, i=1,2, \ldots, l$.

Based on equation (16), we can obtain the conditional probability density function of parameter $\alpha_{i k}, k=0,1$, and 2 :

$$
\begin{aligned}
p\left(\alpha_{i k} \mid \lambda\right) & \propto \alpha_{i k}^{m_{i k}} \alpha_{i}^{m_{i}-m_{i}^{*}} \exp \left\{\lambda_{i}^{-1} \alpha_{i k} A_{i}\right\} \\
& \propto \sum_{q=1}^{m_{i}-m_{i}^{*}} \alpha_{i k}^{m_{i k}+q} \exp \left\{\lambda_{i}^{-1} \alpha_{i k} A_{i}\right\} .
\end{aligned}
$$

When $m_{i}-m_{i}^{*}=0$, the conditional probability density function of parameter $\alpha_{i k}, k=0,1$, and 2, can be expressed as follows:

$$
p\left(\alpha_{i k} \mid \lambda\right) \propto \alpha_{i k}^{m_{i k}} \exp \left\{\lambda_{i}^{-1} \alpha_{i k} A_{i}\right\} .
$$

Then, we can obtain that $p\left(\alpha_{i k} \mid \lambda\right)$ belongs to the gamma distribution family.

Under the condition $\lambda_{i}=\lambda_{i r}$, the condition prior distribution of the parameter $\alpha_{i k}, k=0,1$, and 2 , is Gamma $\left(a_{i k r}, b_{i k r}\right)$, which is the conjugate distribution for the parameter $\alpha_{i k}, k=0,1$, and 2. The PDF can be expressed as follows:

$$
\pi\left(\alpha_{i k} \mid \lambda_{i}=\lambda_{i r}\right)=\frac{d_{i r k}^{c_{i r k}} \alpha_{i k}^{a_{i k}-1} \exp \left(-d_{i k r} \alpha_{i k}\right)}{\Gamma\left(c_{i r k}\right)}
$$

The condition posterior density function of the parameter $\alpha_{i k}, k=0,1$, and 2 , is

$$
\pi\left(\alpha_{i k} \mid t, \lambda_{i}=\lambda_{i r}\right)=\frac{l_{i}(\theta) \pi\left(\alpha_{i k} \mid \lambda_{i}=\lambda_{i r}\right) \pi\left(\lambda_{i}=\lambda_{i r}\right)}{\int l_{i}(\theta) \pi\left(\alpha_{i k} \mid \lambda_{i}=\lambda_{i r}\right) \pi\left(\lambda_{i}=\lambda_{i r}\right) d \alpha_{i k}} .
$$

Using the binomial expansion,

$$
\left(\alpha_{i 0}+\alpha_{i 1}+\alpha_{i 2}\right)^{m_{i}-m_{i}^{*}}=\sum_{u=0}^{m_{i}-m_{i}^{*}}\left(\begin{array}{c}
m_{i}-m_{i}^{*} \\
u
\end{array}\right) \sum_{v=0}^{m_{i}-m_{i}^{*}-u}\left(\begin{array}{c}
m_{i}-m_{i}^{*}-u \\
v
\end{array}\right) \alpha_{i 0}^{u} \alpha_{i 1}^{v} \alpha_{i 2}^{m_{i}-m_{i}^{*}-u-v} .
$$

The likelihood function in equation (15) can be written as the following form:

$$
\begin{aligned}
& l_{i}\left(t \mid \lambda, \alpha_{i 0}, \alpha_{i 1}, \alpha_{i 2}\right) \\
& \propto \sum_{u=0}^{m_{i}-m_{i}^{*}}\left(\begin{array}{c}
m_{i}-m_{i}^{*} \\
u
\end{array}\right) \sum_{v=0}^{m_{i}-m_{i}^{*}-u}\left(\begin{array}{c}
m_{i}-m_{i}^{*}-u \\
v
\end{array}\right) \alpha_{i 0}^{m_{i 0}+u} \alpha_{i 1}^{m_{i 1}+v} \alpha_{i 2}^{m_{i 2}+m_{i}-m_{i}^{*}-u-v} \exp \left\{\lambda_{i} \sum_{j=1}^{m_{i}} t_{i j}-\lambda_{i}^{-1} \alpha_{i} A_{i}\right\} .
\end{aligned}
$$

Then, the condition posterior density function of the parameter $\alpha_{i k}, k=0,1$, and 2, can be obtained as follows:

$$
\begin{aligned}
\pi\left(\alpha_{i 0} \mid \lambda_{i}=\lambda_{i r}, t\right)= & B_{i r}^{-1} \exp \left(-\alpha_{i 0}\left(\lambda_{i r} A_{i r}+d_{i r 0}\right)\right) \\
& \times\left\{\sum_{u=0}^{m_{i}-m_{i}^{*}}\left(\begin{array}{c}
m_{i}-m_{i}^{*} \\
u
\end{array}\right) \sum_{v=0}^{m_{i}-m_{i}^{*}-v}\left(\begin{array}{c}
m_{i}-m_{i}^{*}-u \\
v
\end{array}\right) \frac{\Gamma\left(m_{i 1}+c_{i r 1}+v\right)}{\left(\lambda_{i r} A_{i r}+d_{i r 1}\right)\left(m_{i 1}+c_{i r 1}+v\right)}\right. \\
& \left.\times \frac{\Gamma\left(m_{i 2}+c_{i r 2}+m_{i}-m_{i}^{*}-u-v\right)}{\left(\lambda_{i r} A_{i r}+d_{i r 2}\right)^{\left(m_{i 2}+c_{i r 2}+m_{i}-m_{i}^{*}-u-v\right)} \alpha_{i 0}^{m_{i 0}+c_{i r 0}+u-1}}\right\},
\end{aligned}
$$


8

Mathematical Problems in Engineering

$$
\begin{aligned}
& \pi\left(\alpha_{i 1} \mid \lambda_{i}=\lambda_{i r}, t\right)=B_{i r}^{-1} \exp \left(-\alpha_{i 1}\left(\lambda_{i r} A_{i r}+d_{i r 1}\right)\right) \\
& \times\left\{\sum_{u=0}^{m_{i}-m_{i}^{*}}\left(\begin{array}{c}
m_{i}-m_{i}^{*} \\
u
\end{array}\right) \sum_{v=0}^{m_{i}-m_{i}^{*}-u}\left(\begin{array}{c}
m_{i}-m_{i}^{*}-u \\
v
\end{array}\right) \frac{\Gamma\left(m_{i 0}+c_{i r 0}+u\right)}{\left(\lambda_{i r} A_{i r}+d_{i r 0}\right)^{\left(m_{i 0}+a_{i 0}+u\right)}}\right. \\
& \left.\times \frac{\Gamma\left(m_{i 2}+c_{i r 2}+m_{i}-m_{i}^{*}-u-v\right)}{\left(\lambda_{i r} A_{i r}+d_{i r 2}\right)^{\left(m_{i 2}+a_{i 2}+m_{i}-m_{i}^{*}-u-v\right)}} \alpha_{i 1}^{m_{i 0}+c_{i r 0}+v-1}\right\}, \\
& \pi\left(\alpha_{i 2} \mid t, \lambda_{i}=\lambda_{i r}\right)=B_{i r}^{-1} \exp \left(-\alpha_{i 2}\left(\lambda_{i r} A_{i r}+b_{i r 2}\right)\right) \\
& \times\left\{\sum_{u=0}^{m_{i}-m_{i}^{*}}\left(\begin{array}{c}
m_{i}-m_{i}^{*} \\
u
\end{array}\right) \sum_{v=0}^{m_{i}-m_{i}^{*}-u}\left(\begin{array}{c}
m_{i}-m_{i}^{*}-u \\
v
\end{array}\right) \frac{\Gamma\left(m_{i 0}+a_{i r 0}+u\right)}{\left(\lambda_{i r} A_{i r}+b_{i r 0}\right)^{\left(m_{i 0}+a_{i r 0}+u\right)}}\right. \\
& \left.\times \frac{\Gamma\left(m_{i 1}+a_{i r 1}+v\right)}{\left(\lambda_{i r} A_{i r}+b_{i r 1}\right)^{\left(m_{i 1}+a_{i r 1}+v\right)}} \alpha_{i 2}^{m_{i 2}+a_{i r 2}+m_{i}-m_{i}^{*}-u-v}\right\} \text {, }
\end{aligned}
$$

where $A_{i r}=\sum_{j=1}^{m_{i}}\left(R_{i j}^{*}+1\right)\left(\exp \left(\lambda_{i r} t_{i j}\right)-1\right)$.

The marginal posterior density distribution of the parameter $\lambda_{i}, i=1,2, \ldots, l$, can be obtained as follows:

$$
\begin{aligned}
P_{i r} & =P\left(\lambda_{i}=\lambda_{i r}\right)=\frac{\iiint \prod_{j=1}^{m_{i}} l_{i}\left(t \mid \lambda_{i}, \alpha_{i 0}, \alpha_{i 1}, \alpha_{i 2}\right) \pi\left(\alpha_{i 0}, \alpha_{i 1}, \alpha_{i 2} \mid \lambda_{i}=\lambda_{i r}\right) \pi\left(\lambda_{i}=\lambda_{i r}\right) \mathrm{d} \alpha_{i 0} \mathrm{~d} \alpha_{i 1} \mathrm{~d} \alpha_{i 2}}{\sum_{r=1}^{q_{i}} \iiint \prod_{j=1}^{m_{i}} l_{i}\left(t \mid \lambda_{i}, \alpha_{i 0}, \alpha_{i 1}, \alpha_{i 2}\right) \pi\left(\alpha_{i 0}, \alpha_{i 1}, \alpha_{i 2} \mid \lambda_{i}=\lambda_{i r}\right) \pi\left(\lambda_{i}=\lambda_{i r}\right) \mathrm{d} \alpha_{i 0} \mathrm{~d} \alpha_{i 1} \mathrm{~d} \alpha_{i 2}}, \\
& =\frac{\pi_{i r} \prod_{i=1}^{l}\left(\prod_{k=0}^{2} d_{i r k}^{a_{i r k}} / \Gamma\left(c_{i r k}\right)\right) B_{i r}}{\sum_{r=1}^{q_{i}} \pi_{i r} \prod_{i=1}^{l}\left(\prod_{k=0}^{2} d_{i r k}^{a_{i r k}} / \Gamma\left(c_{i r k}\right)\right) B_{i r}}
\end{aligned}
$$

where

$$
\begin{aligned}
B_{i r}= & \left\{\sum_{u=0}^{m_{i}-m_{i}^{*}}\left(\begin{array}{c}
m_{i}-m_{i}^{*} \\
u
\end{array}\right) \sum_{v=0}^{m_{i}-m_{i}^{*}-u}\left(\begin{array}{c}
m_{i}-m_{i}^{*}-u \\
v
\end{array}\right) \frac{\Gamma\left(m_{i 0}+c_{i r 0}+u\right)}{\left(\lambda_{i r} A_{i r}+d_{i r 0}\right)}\left(m_{i 0}+a_{i r 0}+u\right)\right. \\
& \left.\times \frac{\Gamma\left(m_{i 1}+c_{i r 1}+v\right)}{\left(\lambda_{i r} A_{i r}+d_{i r 1}\right)^{\left(m_{i 1}+c_{i r 1}+v\right)}} \frac{\Gamma\left(m_{i 2}+c_{i r 2}+m_{i}-m_{i}^{*}-u-v\right)}{\left(\lambda_{i r} A_{i r}+d_{i r 2}\right)^{\left(m_{i 2}+c_{i r}+m_{i}-m_{i}^{*}-u-v\right)}}\right\} .
\end{aligned}
$$

Under the squared error loss function, the BEs of $\lambda_{i}, \alpha_{i 0}, \alpha_{i 1}$, and $\alpha_{i 2}$ can be written as follows:

$$
\begin{aligned}
\hat{\lambda}_{i(B S)}= & E^{\lambda_{i} \mid t}\left(\lambda_{i}\right)=\sum_{r=1}^{q_{i}} P_{i r} \lambda_{i r} \\
\hat{\alpha}_{i 0(B S)}= & E^{\alpha_{i 0} \mid \lambda, t}\left(\alpha_{i 0}\right)=\sum_{r=1}^{q_{i}} P_{i r} E\left(\alpha_{i 0}\right)=\sum_{r=1}^{q_{i}} P_{i r} \frac{1}{B_{i r}}\left\{\sum_{u=0}^{m_{i}-m_{i}^{*}}\left(\begin{array}{c}
m_{i}-m_{i}^{*} \\
u
\end{array}\right) \sum_{v=0}^{m_{i}-m_{i}^{*}-u}\left(\begin{array}{c}
m_{i}-m_{i}^{*}-u \\
v
\end{array}\right) \frac{\Gamma\left(m_{i 0}+c_{i r 0}+u+1\right)}{\left(\lambda_{i r} A_{r i}+d_{i r 0}\right)^{\left(m_{i 0}+c_{i r 0}+u+1\right)}}\right. \\
& \left.\times \frac{\Gamma\left(m_{i 1}+c_{i r 1}+v\right)}{\left(\lambda_{i r} A_{r i}+d_{i r 1}\right)^{\left(m_{i 1}+c_{i r 1}+v\right)}} \frac{\Gamma\left(m_{i 2}+c_{i r 2}+m_{i}-m_{i}^{*}-u-v\right)}{\left(\lambda_{i r} A_{i r}+d_{i r 2}\right)^{\left(m_{i 2}+c_{i r 2}+m_{i}-m_{i}^{*}-u-v\right)}}\right\}
\end{aligned}
$$




$$
\begin{aligned}
& \widehat{\alpha}_{i 1(B S)}=E^{\alpha_{i 1} \mid \lambda, t}\left(\alpha_{i 1}\right)=\sum_{r=1}^{q_{i}} P_{i r} E\left(\alpha_{i 1}\right)=\sum_{r=1}^{q_{i}} P_{i r} \frac{1}{B_{i r}}\left\{\sum_{u=0}^{m_{i}-m_{i}^{*}}\left(\begin{array}{c}
m_{i}-m_{i}^{*} \\
u
\end{array}\right)^{m_{i}-m_{i}^{*}-u}\left(\begin{array}{c}
m_{i}-m_{i}^{*}-u \\
v
\end{array}\right) \frac{\Gamma\left(m_{i 0}+c_{i r 0}+u\right)}{\left(\lambda_{i r} A_{r i}+d_{i r 0}\right)\left(m_{i 0}+c_{i r 0}+u\right)}\right. \\
& \left.\times \frac{\Gamma\left(m_{i 1}+c_{i r 1}+v+1\right)}{\left(\lambda_{i r} A_{r i}+d_{i r 1}\right)^{\left(m_{i 1}+c_{i r 1}+v+1\right)}} \frac{\Gamma\left(m_{i 2}+c_{i r 2}+m_{i}-m_{i}^{*}-u-v\right)}{\left(\lambda_{i r} A_{i r}+d_{i r 2}\right)^{\left(m_{i 2}+c_{i r 2}+m_{i}-m_{i}^{*}-u-v\right)}}\right\} \\
& \widehat{\alpha}_{i 2(B S)}=E^{\alpha_{i 2} \mid \lambda, t}\left(\alpha_{i 2}\right)=\sum_{r=1}^{q_{i}} P_{i r} E\left(\alpha_{i 2}\right)=\sum_{r=1}^{q_{i}} P_{i r} \frac{1}{B_{i r}}\left\{\sum_{u=0}^{m_{i}-m_{i}^{*}}\left(\begin{array}{c}
m_{i}-m_{i}^{*} \\
u
\end{array}\right) \sum_{v=0}^{m_{i}-m_{i}^{*}-u}\left(\begin{array}{c}
m_{i}-m_{i}^{*}-u \\
v
\end{array}\right) \frac{\Gamma\left(m_{i 0}+c_{i r 0}+u\right)}{\left(\lambda_{i r} A_{r i}+d_{i r 0}\right)\left(m_{i 0}+c_{i r 0}+u\right)}\right. \\
& \left.\times \frac{\Gamma\left(m_{i 1}+c_{i r 1}+v\right)}{\left(\lambda_{i r} A_{r i}+d_{i r 1}\right)^{\left(m_{i 1}+c_{i r 1}+v\right)}} \frac{\Gamma\left(m_{i 2}+c_{i r 2}+m_{i}-m_{i}^{*}-u-v+1\right)}{\left(\lambda_{i r} A_{i r}+d_{i r 2}\right)^{\left(m_{i 2}+c_{i r 2}+m_{i}-m_{i}^{*}-u-v+1\right)}}\right\} \text {. }
\end{aligned}
$$

3.3. The Estimation of Reliability. The reliability estimation of the unit at any time $t$ under the normal stress level is

$$
\widehat{R}(t)=\exp \left\{-\frac{\left(\widehat{\alpha}_{00}+\widehat{\alpha}_{01}+\widehat{\alpha}_{02}\right)}{\widehat{\lambda}_{0}}\left(\exp \left(\widehat{\lambda}_{0} t\right)-1\right)\right\} .
$$

The estimation of the shape parameter $\lambda_{0}$ under normal stress level $S_{0}$ can be obtained from the equation $\log \lambda_{0}=a+b \varphi\left(S_{0}\right), i=1,2, \ldots, l$. And, the estimation of the scale parameter $\alpha_{0 k}$ under normal stress level $S_{0}$ can be obtained from the equation $\log \alpha_{0 k}=a_{0 k}+b_{0 k} \varphi\left(S_{0}\right)$, $i=1,2, \ldots, l, k=0,1$, and 2 . Putting the estimations of the parameters $\hat{\lambda}_{i}$ and $\widehat{\alpha}_{i k}, i=1,2, \ldots, l, k=0,1$, and 2 , into equations (5) and (6), we can obtain the estimations of $a, b, a_{0 k}$, and $b_{0 k}, k=0,1$, and 2 ,by the least square method:

$$
\begin{gathered}
\widehat{a}=\frac{\sum_{i=1}^{l} \ln \widehat{\lambda}_{i}-\widehat{b} \sum_{i=1}^{l} \varphi\left(S_{i}\right)}{l}, \widehat{b}=\frac{l \sum_{i=1}^{l} \ln \widehat{\lambda}_{i} \varphi\left(S_{i}\right)-\sum_{i=1}^{l} \ln \widehat{\lambda}_{i} \sum_{i=1}^{l} \varphi\left(S_{i}\right)}{l \sum_{i=1}^{l} \varphi^{2}\left(S_{i}\right)-\left(\sum_{i=1}^{l} \varphi\left(S_{i}\right)\right)^{2}}, \\
\widehat{a}_{i k}=\frac{\sum_{i=1}^{l} \ln \widehat{\alpha}_{i k}-\widehat{b}_{i k} \sum_{i=1}^{l} \varphi\left(S_{i}\right)}{l}, \widehat{b}_{i k}=\frac{l \sum_{i=1}^{l} \varphi\left(S_{i}\right) \ln \widehat{\alpha}_{i k}-\sum_{i=1}^{l} \ln \widehat{\alpha}_{i k} \sum_{i=1}^{l} \varphi\left(S_{i}\right)}{l \sum_{i=1}^{l} \varphi^{2}\left(S_{i}\right)-\left(\sum_{i=1}^{l} \varphi\left(S_{i}\right)\right)^{2}} .
\end{gathered}
$$

\section{Interval Estimation}

4.1. Approximation Confidence Intervals. For the parameters $\lambda_{i}$ and $\alpha_{i k}, i=1,2, \ldots, l, k=0,1$, and 2 , it is impossible to get the exact confidence intervals. So, we use the asymptotic property of the MLEs to construct the approximation confidence intervals (ACIs), and the observed Fisher information matrix of the parameters $\lambda_{i}$ and $\alpha_{i k}, i=1,2, \ldots$, $l, k=0,1$, and 2 is denoted by

$$
\widehat{I}(\theta)=\operatorname{diag}\left(\widehat{I}^{1}, \widehat{I}^{2}, \ldots, \widehat{I}^{l}\right),
$$

where the submatrix can be expressed as follows:

$$
\widehat{I}^{i}\left(\lambda_{i}, \alpha_{i 0}, \alpha_{i 1}, \alpha_{i 2}\right)=\left[\begin{array}{llll}
\widehat{I}_{11}^{i} & \widehat{I}_{12}^{i} & \widehat{I}_{13}^{i} & \widehat{I}_{14}^{i} \\
\widehat{I}_{21}^{i} & \widehat{I}_{22}^{i} & \widehat{I}_{23}^{i} & \widehat{I}_{24}^{i} \\
\widehat{I}_{31}^{i} & \widehat{I}_{32}^{i} & \widehat{I}_{33}^{i} & \widehat{I}_{34}^{i} \\
\widehat{I}_{41}^{i} & \widehat{I}_{42}^{i} & \widehat{I}_{43}^{i} & \widehat{I}_{44}^{i}
\end{array}\right],
$$

where

$$
\begin{aligned}
\widehat{I}_{11}^{i}= & -\left.\frac{\partial^{2} \ln L}{\partial \lambda_{i}^{2}}\right|_{\lambda_{i}=\hat{\lambda}_{i}, \alpha=\widehat{\alpha}_{i}}=-\widehat{\lambda}_{i}^{-1} \widehat{\alpha}_{i} \sum_{j=1}^{m_{i}}\left(R_{i j}^{*}+1\right) \exp \left(\widehat{\lambda}_{i} t_{i j}\right) t_{i j}^{2}+2 \widehat{\lambda}_{i}^{-2} \widehat{\alpha}_{i} \sum_{j=1}^{m_{i}}\left(R_{i j}^{*}+1\right) \exp \left(\widehat{\lambda}_{i} t_{i j}\right) t_{i j} \\
& -2 \widehat{\lambda}_{i}^{-3} \widehat{\alpha}_{i} \sum_{j=1}^{m_{i}}\left(R_{i j}^{*}+1\right)\left(\exp \left(\widehat{\lambda}_{i} t_{i j}\right)-1\right), \\
\widehat{I}_{1(k+2)}^{i}= & \widehat{I}_{(k+2) 1}^{i}=-\left.\frac{\partial^{2} \ln L}{\partial \lambda_{i} \partial \alpha_{i k}}\right|_{\lambda_{i}=\hat{\lambda}, \alpha_{i k}=\widehat{\alpha}_{i k}}=-\hat{\lambda}_{i}^{-1} \sum_{j=1}^{m_{i}}\left(R_{i j}^{*}+1\right) \exp \left(\widehat{\lambda}_{i} t_{i j}\right) t_{i j}-\widehat{\lambda}_{i}^{-2} \sum_{j=1}^{m_{i}}\left(R_{i j}^{*}+1\right)\left(\exp \left(\hat{\lambda}_{i} t_{i j}\right)-1\right),
\end{aligned}
$$




$$
\begin{aligned}
\widehat{I}_{(k+2)(k+2)}^{i} & =-\left.\frac{\partial^{2} \ln L}{\partial \alpha_{i k}^{2}}\right|_{\alpha_{i k}=\widehat{\alpha}_{i k}}=\frac{-m_{i k}}{\widehat{a}_{i k}^{2}}+\frac{-\left(m_{i}-m_{i}^{*}\right)}{\widehat{a}_{i}^{2}} \\
\widehat{I}_{23}^{i} & =\widehat{I}_{32}^{i}=\widehat{I}_{24}^{i}=\widehat{I}_{42}^{i}=\widehat{I}_{34}^{i}=\widehat{I}_{43}^{i}=-\left.\frac{\partial^{2} \ln L}{\partial \alpha_{i k} \partial \alpha_{i h}}\right|_{\alpha_{i k}=\widehat{\alpha}_{i k}, \alpha_{i h}=\widehat{\alpha}_{i h}}=-\frac{\left(m_{i}-m_{i}^{*}\right)}{\widehat{\alpha}_{i}^{2}}, \quad k \text { and } h=0,1, \text { and } 2, k \neq h .
\end{aligned}
$$

The variance-covariance matrix of the parameters $\lambda_{i}$ and $\alpha_{i k}, i=1,2, \ldots, l, k=0,1$, and 2 , can be obtained as follows:

$$
\widehat{V}(\theta)=(\widehat{I}(\theta))^{-1}=\operatorname{diag}\left(\left(\widehat{I}^{1}\right)^{-1},\left(\widehat{I}^{2}\right)^{-1}, \ldots,\left(\widehat{I}^{l}\right)^{-1}\right) .
$$

Let

$$
\begin{aligned}
\widehat{V}^{i}\left(\lambda_{i}, \alpha_{i 0}, \alpha_{i 1}, \alpha_{i 2}\right) & =\left(\widehat{I}^{i}\left(\lambda_{i}, \alpha_{i 0}, \alpha_{i 1}, \alpha_{i 2}\right)\right)^{-1} \\
& =\left[\begin{array}{cccc}
\widehat{V}_{11}^{i} & \widehat{V}_{12}^{i} & \widehat{V}_{13}^{i} & \widehat{V}_{14}^{i} \\
\widehat{V}_{21}^{i} & \widehat{V}_{22}^{i} & \widehat{V}_{23}^{i} & \widehat{V}_{24}^{i} \\
\widehat{V}_{31}^{i} & \widehat{V}_{32}^{i} & \widehat{V}_{33}^{i} & \widehat{V}_{34}^{i} \\
\widehat{V}_{41}^{i} & \widehat{V}_{42}^{i} & \widehat{V}_{43}^{i} & \widehat{V}_{44}^{i}
\end{array}\right] \\
& =\left[\begin{array}{cccc}
\widehat{I}_{11}^{i} & \widehat{I}_{12}^{i} & \widehat{I}_{13}^{i} & \widehat{I}_{14}^{i} \\
\widehat{I}_{21}^{i} & \widehat{I}_{22}^{i} & \widehat{I}_{23}^{i} & \widehat{I}_{24}^{i} \\
\widehat{I}_{31}^{i} & \widehat{I}_{32}^{i} & \widehat{I}_{33}^{i} & \widehat{I}_{34}^{i} \\
\widehat{I}_{41}^{i} & \widehat{I}_{42}^{i} & \widehat{I}_{43}^{i} & \widehat{I}_{44}^{i}
\end{array}\right]^{-1}
\end{aligned}
$$

For the given confidence level $\gamma$, the $100(1-\gamma) \%$ ACIs of parameters $\lambda_{i}$ and $\alpha_{i k}, k=0,1$, and 2 , can be expressed as follows:

$$
\begin{aligned}
&\left(\hat{\lambda}_{i}^{L}, \hat{\lambda}_{i}^{U}\right)=\left[\hat{\lambda}_{i}-Z_{\gamma / 2} \sqrt{\widehat{V}_{11}^{i}}, \hat{\lambda}_{i}+Z_{\gamma / 2} \sqrt{\widehat{V}_{11}^{i}}\right], \\
&\left(\hat{\alpha}_{i k}^{L}, \widehat{\alpha}_{i k}^{U}\right)=\left[\widehat{\alpha}_{i k}-Z_{\gamma / 2} \sqrt{\widehat{V}_{(k+1)(k+1)}^{i}}, \widehat{\alpha}_{i k}+Z_{\gamma / 2} \sqrt{\widehat{V}_{(k+1)(k+1)}^{i}}\right], \\
& k=0,1,2,
\end{aligned}
$$

where $Z_{\gamma / 2}$ is the upper $\gamma / 2$ percentile point of the standard normal distribution.

4.2. Highest Posterior Density Credible Intervals. The $100(1-$ $\gamma) \%$ two-side Bayes credible interval (BCI) of $\alpha_{i 0}$ which is denoted by $\left[\hat{\alpha}_{i 0}^{B L}, \widehat{\alpha}_{i 0}^{B U}\right]$ can be obtained by solving the following two equations:

$$
\begin{gathered}
\frac{\gamma}{2}=\int_{0}^{\hat{\alpha}_{i 0}^{B L}} \pi\left(\alpha_{i 0} \mid \lambda, t\right) \mathrm{d} \alpha_{i 0}, \\
1-\frac{\gamma}{2}=\int_{0}^{\widehat{\alpha}_{i 0}^{B U}} \pi\left(\alpha_{i 0} \mid \lambda, t\right) \mathrm{d} \alpha_{i 0} .
\end{gathered}
$$

Substituting equation (46) into (60) and (61), we have

$$
\begin{aligned}
& \gamma / 2=\frac{1}{B_{i}}\left\{\sum_{u=0}^{m_{i}-m_{i}^{*}}\left(\begin{array}{c}
m_{i}-m_{i}^{*} \\
u
\end{array}\right) \sum_{v=0}^{m_{i}-m_{i}^{*}-u}\left(\begin{array}{c}
m_{i}-m_{i}^{*}-u \\
v
\end{array}\right) \Gamma\left(m_{i 1}+c_{i 1}+v\right)\right. \\
& \left.\times \Gamma\left(m_{i 2}+c_{i 2}+m_{i}-m_{i}^{*}-u-v\right) C_{u v}\left(\lambda_{i} A_{i}+d_{i 0}\right)^{-\left(m_{i 0}+c_{i 0}+u\right)} \times \Gamma\left(m_{i 0}+c_{i 0}+u,\left(\lambda_{i} A_{i}+d_{i 0}\right) \widehat{\alpha}_{i 0}^{B L}\right)\right\}, \\
& 1-\frac{\gamma}{2}=\frac{1}{B_{i}}\left\{\sum_{u=0}^{m_{i}-m_{i}^{*}}\left(\begin{array}{c}
m_{i}-m_{i}^{*} \\
u
\end{array}\right) \sum_{v=0}^{m_{i}-m_{i}^{*}-u}\left(\begin{array}{c}
m_{i}-m_{i}^{*}-u \\
v
\end{array}\right) \Gamma\left(m_{i 1}+c_{i 1}+v\right)\right. \\
& \left.\times \Gamma\left(m_{i 2}+c_{i 2}+m_{i}-m_{i}^{*}-u-v\right) C_{u v}\left(\lambda_{i} A_{i}+d_{i 0}\right)^{-\left(m_{i 0}+c_{i 0}+u\right)} \times \Gamma\left(m_{i 0}+c_{i 0}+u,\left(\lambda_{i} A_{i}+d_{i 0}\right) \widehat{\alpha}_{i 0}^{B U}\right)\right\},
\end{aligned}
$$

where

$$
\begin{aligned}
C_{u v}= & \left(\lambda_{i} A_{i}+d_{i 0}\right)^{\left(m_{i 0}+c_{i 0}+u\right)}\left(\lambda_{i} A_{i}+d_{i 1}\right)^{\left(m_{i 1}+c_{i 1}+v\right)} \\
& \cdot\left(\lambda_{i} A_{i}+d_{i 2}\right)^{\left(m_{i 2}+c_{i 2}+m_{i}-m_{i}^{*}-u-v\right)} .
\end{aligned}
$$

And, $\Gamma(a, x)=\int_{0}^{x} t^{a-1} e^{-t} \mathrm{~d} t$ is an incomplete gamma function.
Equations (62) and (63) cannot yield the explicit solutions for $\widehat{\alpha}_{i 0}^{B L}$ and $\widehat{\alpha}_{i 0}^{B U}$. Thus, we can only obtain the numerical solution. Similarly, we can get $\left[\widehat{\alpha}_{i k}^{B L}, \widehat{\alpha}_{i k}^{B U}\right], k=1$ and 2 .

When $m_{i}-m_{i}^{*}=0$, the parameter $\alpha_{i k}, k=0,1$, and 2, follows the gamma distribution with parameters $m_{i k}+c_{i k}$ and $\lambda_{i} A_{i}+d_{i k}$, and it is denoted by $\alpha_{i k} \sim \operatorname{Gamma}\left(m_{i k}+\right.$ $\left.c_{i k}, \lambda_{i} A_{i}+d_{i k}\right), k=0,1$, and 2. The $100(1-\gamma) \%$ two-side 
BCI of $\alpha_{i k}, k=0,1$, and 2, can be acquired by the following equations:

$$
\begin{gathered}
\frac{\gamma}{2}=\int_{0}^{\hat{\alpha}_{i k}^{B U}} \frac{\left(\lambda_{i} A_{i}+d_{i k}\right)^{\left(m_{i k}+c_{i k}\right)}}{\Gamma\left(m_{i k}+c_{i k}\right)} \alpha_{i k}^{m_{i k}+c_{i k}-1} \exp \left(-\alpha_{i k}\left(\lambda_{i} A_{i}+d_{i k}\right)\right) \mathrm{d} \alpha_{i k}, \\
1-\frac{\gamma}{2}=\int_{0}^{\hat{\alpha}_{i k}} \frac{\left(\lambda_{i} A_{i}+d_{i k}\right)^{\left(m_{i k}+c_{i k}\right)}}{\Gamma\left(m_{i k}+c_{i k}\right)} \alpha_{i k}^{m_{i k}+c_{i k}-1} \exp \left(-\alpha_{i k}\left(\lambda_{i} A_{i}+d_{i k}\right)\right) \mathrm{d} \alpha_{i k} .
\end{gathered}
$$

In this case, if $\alpha_{i k}, k=0,1$, and 2, is an integer, the $100(1-\gamma) \% \mathrm{BCI}$ is

$$
\left[\frac{\chi_{1-\gamma / 2}^{2}\left(2\left(m_{i k}+c_{i k}\right)\right)}{2\left(\lambda_{i} A_{i}+d_{i k}\right)}, \frac{\chi_{\gamma / 2}^{2}\left(2\left(m_{i k}+c_{i k}\right)\right)}{2\left(\lambda_{i} A_{i}+d_{i k}\right)}\right] .
$$

The highest posterior density (HPD) CIs of $\alpha_{i k}, k=0,1$, and 2, say $\left[\hat{\alpha}_{i k}^{H L}, \hat{\alpha}_{i k}^{H U}\right]$, is the shortest interval and can be obtained from $\hat{\alpha}_{i k}^{H L}-\hat{\alpha}_{i k}^{H U}=\min \left(\hat{\alpha}_{i k}^{B L}-\hat{\alpha}_{i k}^{B U}\right), k=0,1$, and 2 .

\section{Numerical Simulation}

5.1. Simulation Study. Consider a two-level CSALT under AT-II PHCS. The subjected units have two dependent failure causes where the lifetime of the two failure causes are assumed to follow a MOBG distribution. Suppose the temperature is the accelerate stress, the accelerated stress levels are $S_{1}=50^{\circ} \mathrm{C}=323 \mathrm{~K}$ and $S_{2}=80^{\circ} \mathrm{C}=353 \mathrm{~K}$, and the normal stress level is $S_{0}=25^{\circ} \mathrm{C}=298 \mathrm{~K}$.

Under the stress level $S_{i}, i=1$ and 2, the sample size is $n_{1}=n_{2}=30,40$, and 50, and the predetermined failure numbers are $m_{1}=(10,15,20,25)$ and $m_{2}=(15,20,25,30)$. The life tests are allowed to run over the time $\tau=(0.5,1)$. It is assumed that the proportion of failure data which has unknown failure cause is $10 \%$. Three different progressive censoring schemes are used in the CSALT, which are denoted as follows.

$$
\begin{aligned}
& \text { Scheme 1: } R_{1}=R_{2}=\cdots=R_{m-1}=0 \text { and } R_{m}=n-m \\
& \text { Scheme 2: } R_{1}=n-m \text { and } R_{2}=\cdots=R_{m-1}=R_{m}=0 \\
& \text { Scheme 3: } R_{1}=R_{2}=\cdots=R_{[m / 2]}=1, R_{[(m+1) / 2]}=\cdots= \\
& R_{m-1}=0 \text { and } R_{m}=n-m-[m / 2]
\end{aligned}
$$

The parameters $\lambda_{i}, \alpha_{i 0}, \alpha_{i 1}$, and $\alpha_{i 2}$ are given by the accelerated life functions as follows:

$$
\begin{aligned}
\log \lambda_{i} & =-6+1500 / s_{i}, \quad \log a_{i 0}=-3+800 / s_{i}, \\
\log a_{i 1} & =-4+1000 / s_{i}, \log a_{i 2}=-4+1200 / s_{i} .
\end{aligned}
$$

Then, the initial values of the parameters are $\left(\lambda_{1}, \alpha_{10}\right.$, $\left.\alpha_{11}, \alpha_{12}\right)=(0.2577,0.5926,0.4049,0.7521)$ and $\left(\lambda_{2}, \alpha_{20}, \alpha_{21}\right.$, $\left.\alpha_{22}\right)=(0.1733,0.4801,0.3113,0.5484)$. To compute the BEs and HPD CIs of the parameters, the hyperparameters of the priors are given by $\left(c_{i 0}, c_{i 1}, c_{i 2}\right)=(1,1,3)$ and $\left(d_{i 0}, d_{i 1}, d_{i 2}\right)=$ $(1,1,1)$. The prior distribution of $\lambda_{i}, i=1$ and 2 , is given in Table 1.
Under different progressive censoring schemes, the process is repeated 1000 times, and then, the expected values of MLEs and BEs and mean squared errors (MSEs) of the unknown parameters are computed, and the results are shown in Tables 2 and 3. The average interval lengths (AIL) and coverage percentages (CPs) of ACIs and HPD CIs with the given significant level (0.05) are also computed. The results are shown in Tables 4 and 5 .

From Tables 2 and 3, it can be observed that the MSEs of the MLEs and BEs for the unknown parameters are decreased with the sample size $n$ increase or the effective sample size $m$ increases, and the MLEs and BEs of the unknown parameters are closer to the true values. For the bigger $\tau$, the MSEs for the MLEs and Bayes of unknown parameters both are the smaller. In addition, the difference under three difference progressive censored schemes is not sensible. Furthermore, the BEs of the unknown parameters are better than MLEs, and its MSEs are always less than the MLEs.

From Tables 4 and 5, the CPs of ACIs and HPD CIs for the unknown parameters are increased, and meanwhile, the AILs of the ACIs and the HPD CIs are decreased with the sample size $n$ or the effective sample size $m$ increase. For the fixed $n, m$, and $\tau$, the CPs of HPD CIs are more stable than the ACIs for the unknown parameters.

5.2. Real Data Analysis. To illustrate the proposed methods, a real data is selected from Ebrahimi [25], of which the accelerated stress is voltage, the stress levels are $S_{1}=10 \mathrm{~V}$, $S_{2}=15 \mathrm{~V}$, and $S_{3}=20 \mathrm{~V}$, and the normal stress level is $S_{0}=5 \mathrm{~V}$. Furthermore, for each stress level, the life tests are allowed to run over the time $\left(\tau_{1}, \tau_{2}, \tau_{3}\right)=(2,1.5,1)$, the numbers under the test were $\left(n_{1}, n_{2}, n_{3}\right)=(15,15,15)$, the expected failure number are $\left(m_{1}, m_{2}, m_{3}\right)=(11,12,13)$, and the progressive censoring scheme is $R_{1}=R_{2}=\cdots=R_{m_{i}-1}=$ 0 and $R_{m_{i}}=n_{i}-m_{i}, i=1,2$, and 3 .

Assume that the unknown failure cause of the failure data proportion is $20 \%$, and then, the failure data and the corresponding failure causes under each stress level are presented in Table 6.

For the Bayes analysis, we select small values of the hyperparameters $\quad c_{i 0}=c_{i 1}=c_{i 2}=d_{i 0}=d_{i 1}=d_{i 2}=0.0001$, which gives noninformation priors [26]. We make the data in Table 6 be brought to the above mode and obtain the point estimation and interval estimation of the unknown parameters. The results are shown in Table 7. 
TABLE 1: The prior distribution of the parameter $\lambda_{i}, i=1$ and 2 .

\begin{tabular}{lllcrr}
\hline$\lambda_{1}$ & 0.2 & 0.3 & $\lambda_{2}$ & 0.15 & 0.2 \\
\hline$P\left(\lambda_{1}=\lambda_{1 r}\right)$ & $1 / 2$ & $1 / 2$ & $P\left(\lambda_{2}=\lambda_{2 r}\right)$ & $1 / 2$ & $1 / 2$ \\
\hline
\end{tabular}

TABLE 2: The MLEs and MSEs for the unknown parameters $(\tau=0.5)$.

\begin{tabular}{|c|c|c|c|c|c|c|c|c|c|c|}
\hline$\left(n, m_{1}, m_{2}\right)$ & Scheme & $\begin{array}{l}\text { Estimator } \\
\text { (MSE) }\end{array}$ & $\lambda_{1}$ & $\alpha_{10}$ & $\alpha_{11}$ & $\alpha_{12}$ & $\lambda_{2}$ & $\alpha_{20}$ & $\alpha_{21}$ & $\alpha_{22}$ \\
\hline \multirow{12}{*}{$(30,10,15)$} & \multirow{4}{*}{1} & \multirow{2}{*}{ MLE } & 0.3702 & 0.7014 & 0.5158 & 0.8842 & 0.2689 & 0.5969 & 0.4376 & 0.6534 \\
\hline & & & $(0.0998)$ & $(0.1089)$ & $(0.1067)$ & $(0.1274)$ & $(0.0873)$ & $(0.1081)$ & $(0.0967)$ & $(0.1093)$ \\
\hline & & \multirow{2}{*}{ Bayes } & 0.3498 & 0.6912 & 0.5036 & 0.8775 & 0.2517 & 0.5784 & 0.4128 & 0.6232 \\
\hline & & & $(0.0884)$ & $(0.0987)$ & $(0.0957)$ & $(0.1054)$ & $(0.0782)$ & $(0.0975)$ & $(0.0897)$ & $(0.0972)$ \\
\hline & \multirow{4}{*}{2} & \multirow{2}{*}{ MLE } & 0.3668 & 0.7012 & 0.5178 & 0.8834 & 0.2687 & 0.5975 & 0.4377 & 0.6513 \\
\hline & & & $(0.0987)$ & $(0.1047)$ & $(0.1017)$ & $(0.1276)$ & $(0.0879)$ & $(0.1077)$ & $(0.0999)$ & (0.1099) \\
\hline & & \multirow{2}{*}{ Bayes } & 0.3484 & 0.6841 & 0.4878 & 0.6279 & 0.2512 & 0.5765 & 0.4086 & 0.6235 \\
\hline & & & $(0.0825)$ & $(0.0989)$ & $(0.0935)$ & $(0.1053)$ & $(0.0785)$ & $(0.0978)$ & $(0.0899)$ & $(0.0974)$ \\
\hline & \multirow{4}{*}{3} & \multirow{2}{*}{ MLE } & 0.3677 & 0.7005 & 0.5175 & 0.8832 & 0.2669 & 0.5999 & 0.4372 & 0.6511 \\
\hline & & & $(0.1017)$ & $(0.1089)$ & $(0.1019)$ & $(0.1259)$ & $(0.0957)$ & $(0.1076)$ & $(0.0972)$ & $(0.1075)$ \\
\hline & & \multirow{2}{*}{ Bayes } & 0.3489 & 0.6817 & 0.4976 & 0.8536 & 0.2511 & 0.5766 & 0.4095 & 0.6231 \\
\hline & & & $(0.0822)$ & $(0.0988)$ & $(0.0932)$ & $(0.1061)$ & $(0.0791)$ & $(0.0977)$ & $(0.0895)$ & (0.0979) \\
\hline \multirow{12}{*}{$(30,15,20)$} & \multirow{4}{*}{1} & \multirow{2}{*}{ MLE } & 0.3515 & 0.6895 & 0.4996 & 0.6333 & 0.2574 & 0.5873 & 0.4232 & 0.4513 \\
\hline & & & $(0.0879)$ & $(0.0992)$ & $(0.0972)$ & $(0.1162)$ & $(0.0783)$ & $(0.0989)$ & $(0.0891)$ & $(0.0978)$ \\
\hline & & \multirow{2}{*}{ Bayes } & 0.3378 & 0.6789 & 0.4879 & 0.8489 & 0.2486 & 0.5703 & 0.4036 & 0.6177 \\
\hline & & & $(0.0795)$ & $(0.0907)$ & $(0.0889)$ & $(0.0992)$ & $(0.0699)$ & $(0.0838)$ & $(0.0817)$ & $(0.0897)$ \\
\hline & & \multirow{2}{*}{ MLE } & 0.3484 & 0.6884 & 0.5018 & 0.8699 & 0.2543 & 0.5884 & 0.4259 & 0.6417 \\
\hline & 2 & & $(0.0875)$ & $(0.0992)$ & $(0.0977)$ & $(0.1164)$ & $(0.0791)$ & $(0.0985)$ & $(0.0888)$ & $(0.0981)$ \\
\hline & 2 & \multirow{2}{*}{ Bayes } & 0.3355 & 0.6747 & 0.4857 & 0.8481 & 0.2453 & 0.5654 & 0.4003 & 0.6158 \\
\hline & & & $(0.0784)$ & $(0.0906)$ & $(0.0888)$ & $(0.0987)$ & $(0.0697)$ & $(0.0832)$ & $(0.0818)$ & $(0.0894)$ \\
\hline & \multirow{4}{*}{3} & \multirow{2}{*}{ MLE } & 0.3477 & 0.6876 & 0.5024 & 0.8696 & 0.2565 & 0.5886 & 0.4257 & 0.6413 \\
\hline & & & $(0.0878)$ & $(0.0998)$ & $(0.0975)$ & $(0.1164)$ & $(0.0787)$ & $(0.0997)$ & $(0.0895)$ & $(0.0982)$ \\
\hline & & \multirow{2}{*}{ Bayes } & 0.3355 & 0.6725 & 0.4787 & 0.8452 & 0.2457 & 0.5655 & 0.3988 & 0.6139 \\
\hline & & & $(0.0794)$ & $(0.0905)$ & $(0.0884)$ & $(0.0987)$ & $(0.0695)$ & $(0.0844)$ & $(0.0819)$ & $(0.0893)$ \\
\hline & & & 0.3379 & 0.6712 & 0.4916 & 0.8559 & 0.2416 & 0.5753 & 0.4118 & 0.6313 \\
\hline & & MLE & $(0.0816)$ & $(0.0939)$ & $(0.0871)$ & $(0.1027)$ & $(0.0725)$ & $(0.0894)$ & $(0.0829)$ & $(0.0882)$ \\
\hline & 1 & Bayes & 0.3255 & 0.6689 & 0.4685 & 0.8354 & 0.2398 & 0.5596 & 0.3975 & 0.6166 \\
\hline & & Dayes & $(0.0711)$ & $(0.0847)$ & $(0.0768)$ & $(0.0915)$ & $(0.0604)$ & $(0.0775)$ & $(0.0753)$ & $(0.0811)$ \\
\hline & & MIF & 0.337 & 0.6775 & 0.4886 & 0.8594 & 0.2487 & 0.5764 & 0.4119 & 0.6327 \\
\hline & & MLE & $(0.0808)$ & $(0.0936)$ & $(0.0883)$ & $(0.1031)$ & $(0.0727)$ & $(0.0881)$ & $(0.0831)$ & $(0.0871)$ \\
\hline$(40$ & 2 & & 0.3273 & 0.6672 & 0.4694 & 0.8376 & 0.2369 & 0.5581 & 0.3876 & 0.6165 \\
\hline & & Bayes & $(0.0716)$ & $(0.0852)$ & $(0.0769)$ & $(0.0917)$ & $(0.0609)$ & $(0.0772)$ & $(0.0772)$ & $(0.0817)$ \\
\hline & & & 0.3378 & 0.6786 & 0.4878 & 0.8593 & 0.2468 & 0.5763 & 0.4101 & 0.6368 \\
\hline & 3 & MLE & $(0.0816)$ & $(0.0932)$ & $(0.0894)$ & $(0.1029)$ & $(0.0728)$ & $(0.0895)$ & $(0.0821)$ & $(0.0897)$ \\
\hline & 3 & & 0.3288 & 0.6691 & 0.4691 & 0.8378 & 0.2371 & 0.5568 & 0.3893 & 0.6045 \\
\hline & & Bayes & $(0.0714)$ & $(0.0859)$ & $(0.0762)$ & $(0.0919)$ & $(0.0612)$ & $(0.0778)$ & $(0.0768)$ & $(0.0813)$ \\
\hline & & & 0.325 & 0.6611 & 0.4768 & 0.8438 & 0.2359 & 0.5667 & 0.3964 & 0.6269 \\
\hline & 1 & MLE & $(0.0764)$ & $(0.0849)$ & $(0.0779)$ & $(0.0945)$ & $(0.0679)$ & $(0.0812)$ & $(0.0785)$ & $(0.0811)$ \\
\hline & 1 & & 0.3143 & 0.6571 & 0.4623 & 0.8294 & 0.2252 & 0.5508 & 0.3758 & 0.6044 \\
\hline & & Bayes & $(0.0656)$ & $(0.0776)$ & $(0.0682)$ & $(0.0897)$ & $(0.0587)$ & $(0.0705)$ & $(0.0711)$ & $(0.0732)$ \\
\hline & & & 0.3255 & 0.669 & 0.4758 & 0.8493 & 0.2375 & 0.5674 & 0.4044 & 0.6277 \\
\hline$(40-2025)$ & 2 & ML & $(0.0762)$ & $(0.0843)$ & $(0.0771)$ & $(0.0951)$ & $(0.0682)$ & $(0.0815)$ & $(0.0794)$ & $(0.0809)$ \\
\hline$(40,20,25)$ & 2 & & 0.3155 & 0.6549 & 0.4589 & 0.8283 & 0.2275 & 0.5496 & 0.3779 & 0.6036 \\
\hline & & Bayes & $(0.0657)$ & $(0.0779)$ & $(0.0687)$ & $(0.0892)$ & $(0.0583)$ & $(0.0702)$ & $(0.0713)$ & $(0.0735)$ \\
\hline & & & 0.3235 & 0.6673 & 0.4686 & 0.8392 & 0.2388 & 0.5647 & 0.3988 & 0.6249 \\
\hline & 3 & MLF & $(0.0764)$ & $(0.0858)$ & $(0.0773)$ & $(0.0953)$ & $(0.0681)$ & $(0.0811)$ & $(0.0789)$ & $(0.0809)$ \\
\hline & 3 & & 0.3153 & 0.6585 & 0.4578 & 0.8254 & 0.2369 & 0.5486 & 0.3764 & 0.5984 \\
\hline & & Bayes & $(0.0653)$ & $(0.0778)$ & $(0.0684)$ & $(0.0893)$ & $(0.0585)$ & $(0.0708)$ & $(0.0712)$ & $(0.0736)$ \\
\hline
\end{tabular}


TABle 2: Continued.

\begin{tabular}{|c|c|c|c|c|c|c|c|c|c|c|}
\hline$\left(n, m_{1}, m_{2}\right)$ & Scheme & $\begin{array}{l}\text { Estimator } \\
\text { (MSE) }\end{array}$ & $\lambda_{1}$ & $\alpha_{10}$ & $\alpha_{11}$ & $\alpha_{12}$ & $\lambda_{2}$ & $\alpha_{20}$ & $\alpha_{21}$ & $\alpha_{22}$ \\
\hline \multirow{7}{*}{$(50,20,25)$} & \multirow[b]{2}{*}{1} & MLE & $\begin{array}{c}0.3053 \\
(0.0635)\end{array}$ & $\begin{array}{c}0.6538 \\
(0.0758)\end{array}$ & $\begin{array}{c}0.4655 \\
(0.0661)\end{array}$ & $\begin{array}{c}0.8281 \\
(0.0863)\end{array}$ & $\begin{array}{c}0.2268 \\
(0.0584)\end{array}$ & $\begin{array}{c}0.5466 \\
(0.0718)\end{array}$ & $\begin{array}{c}0.3861 \\
(0.0681)\end{array}$ & $\begin{array}{c}0.6129 \\
(0.0711)\end{array}$ \\
\hline & & Bayes & $\begin{array}{c}0.2944 \\
(0.0558)\end{array}$ & $\begin{array}{c}0.6406 \\
(0.0675)\end{array}$ & $\begin{array}{c}0.4438 \\
(0.0578)\end{array}$ & $\begin{array}{c}0.8192 \\
(0.0783)\end{array}$ & $\begin{array}{c}0.2181 \\
(0.0511)\end{array}$ & $\begin{array}{c}0.5324 \\
(0.0625)\end{array}$ & $\begin{array}{c}0.3666 \\
(0.0609)\end{array}$ & $\begin{array}{c}0.5893 \\
(0.0633)\end{array}$ \\
\hline & \multirow{3}{*}{2} & MLE & $\begin{array}{c}0.3049 \\
(0.0631)\end{array}$ & $\begin{array}{c}0.6596 \\
(0.0757)\end{array}$ & $\begin{array}{c}0.4739 \\
(0.0663)\end{array}$ & $\begin{array}{c}0.8295 \\
(0.0859)\end{array}$ & $\begin{array}{c}0.2254 \\
(0.0587)\end{array}$ & $\begin{array}{c}0.5542 \\
(0.0714)\end{array}$ & $\begin{array}{c}0.3913 \\
(0.0682)\end{array}$ & $\begin{array}{c}0.6128 \\
(0.0717)\end{array}$ \\
\hline & & & 0.2963 & 0.6393 & 0.4475 & 0.8186 & 0.2188 & 0.5337 & 0.3651 & 0.585 \\
\hline & & Bayes & $(0.0554)$ & $(0.0681)$ & $(0.0576)$ & $(0.0787)$ & $(0.0513)$ & $(0.0626)$ & $(0.0607)$ & $(0.0631)$ \\
\hline & \multirow[b]{2}{*}{3} & MLE & $\begin{array}{c}0.3099 \\
(0.0633)\end{array}$ & $\begin{array}{c}0.6545 \\
(0.0754)\end{array}$ & $\begin{array}{c}0.4669 \\
(0.0664)\end{array}$ & $\begin{array}{c}0.8288 \\
(0.0862)\end{array}$ & $\begin{array}{c}0.2283 \\
(0.0586)\end{array}$ & $\begin{array}{c}0.5546 \\
(0.0716)\end{array}$ & $\begin{array}{c}0.3889 \\
(0.0683)\end{array}$ & $\begin{array}{c}0.6137 \\
(0.0719)\end{array}$ \\
\hline & & Bayes & $\begin{array}{c}0.2989 \\
(0.0552)\end{array}$ & $\begin{array}{c}0.6379 \\
(0.0678)\end{array}$ & $\begin{array}{c}0.4461 \\
(0.0575)\end{array}$ & $\begin{array}{c}0.8187 \\
(0.0785)\end{array}$ & $\begin{array}{c}0.2185 \\
(0.059)\end{array}$ & $\begin{array}{c}0.5348 \\
(0.0624)\end{array}$ & $\begin{array}{c}0.3666 \\
(0.0608)\end{array}$ & $\begin{array}{c}0.5881 \\
(0.0632)\end{array}$ \\
\hline \multirow{6}{*}{$(50,25,30)$} & \multirow{2}{*}{1} & MLE & $\begin{array}{c}0.2892 \\
(0.0512)\end{array}$ & $\begin{array}{c}0.6438 \\
(0.0642)\end{array}$ & $\begin{array}{c}0.4551 \\
(0.0518)\end{array}$ & $\begin{array}{c}0.8189 \\
(0.0778)\end{array}$ & $\begin{array}{c}0.2164 \\
(0.0487)\end{array}$ & $\begin{array}{c}0.5377 \\
(0.0614)\end{array}$ & $\begin{array}{c}0.3747 \\
(0.0528)\end{array}$ & $\begin{array}{c}0.6085 \\
(0.0684)\end{array}$ \\
\hline & & Bayes & $\begin{array}{c}0.2876 \\
(0.0419)\end{array}$ & $\begin{array}{c}0.6368 \\
(0.0565)\end{array}$ & $\begin{array}{c}0.4329 \\
(0.0509)\end{array}$ & $\begin{array}{c}0.8029 \\
(0.0695)\end{array}$ & $\begin{array}{c}0.2089 \\
(0.0409)\end{array}$ & $\begin{array}{c}0.5248 \\
(0.0512)\end{array}$ & $\begin{array}{c}0.3586 \\
(0.0482)\end{array}$ & $\begin{array}{c}0.5784 \\
(0.0537)\end{array}$ \\
\hline & \multirow{2}{*}{2} & MLE & $\begin{array}{c}0.2902 \\
(0.0511)\end{array}$ & $\begin{array}{c}0.6437 \\
(0.0639)\end{array}$ & $\begin{array}{c}0.4548 \\
(0.0517)\end{array}$ & $\begin{array}{c}0.8173 \\
(0.0776)\end{array}$ & $\begin{array}{c}0.2174 \\
(0.0485)\end{array}$ & $\begin{array}{c}0.5393 \\
(0.0618)\end{array}$ & $\begin{array}{c}0.3773 \\
(0.0527)\end{array}$ & $\begin{array}{c}0.6072 \\
(0.0681)\end{array}$ \\
\hline & & Bayes & $\begin{array}{c}0.2851 \\
(0.0411)\end{array}$ & $\begin{array}{c}0.6369 \\
(0.0567)\end{array}$ & $\begin{array}{c}0.4356 \\
(0.0502)\end{array}$ & $\begin{array}{c}0.8063 \\
(0.0697)\end{array}$ & $\begin{array}{c}0.2074 \\
(0.0408)\end{array}$ & $\begin{array}{c}0.5245 \\
(0.0513)\end{array}$ & $\begin{array}{c}0.3592 \\
(0.0485)\end{array}$ & $\begin{array}{c}0.5788 \\
(0.0535)\end{array}$ \\
\hline & \multirow[b]{2}{*}{3} & MLE & $\begin{array}{c}0.2874 \\
(0.0513)\end{array}$ & $\begin{array}{c}0.6487 \\
(0.0641)\end{array}$ & $\begin{array}{c}0.4548 \\
(0.0514)\end{array}$ & $\begin{array}{c}0.8186 \\
(0.0777)\end{array}$ & $\begin{array}{c}0.2187 \\
(0.0488)\end{array}$ & $\begin{array}{c}0.5383 \\
(0.0612)\end{array}$ & $\begin{array}{c}0.3773 \\
(0.0525)\end{array}$ & $\begin{array}{c}0.6087 \\
(0.0686)\end{array}$ \\
\hline & & Bayes & $\begin{array}{c}0.2736 \\
(0.0418)\end{array}$ & $\begin{array}{c}0.6295 \\
(0.0561)\end{array}$ & $\begin{array}{c}0.4373 \\
(0.0508)\end{array}$ & $\begin{array}{c}0.8059 \\
(0.0695)\end{array}$ & $\begin{array}{c}0.2077 \\
(0.0411)\end{array}$ & $\begin{array}{c}0.5259 \\
(0.0516)\end{array}$ & $\begin{array}{c}0.3603 \\
(0.0484)\end{array}$ & $\begin{array}{c}0.5778 \\
(0.0536)\end{array}$ \\
\hline
\end{tabular}

TABLE 3: The MLEs and MSEs for the unknown parameters $(\tau=1)$.

\begin{tabular}{|c|c|c|c|c|c|c|c|c|c|c|}
\hline$\left(n, m_{1}, m_{2}\right)$ & Scheme & $\begin{array}{c}\text { Estimator } \\
\text { (MSE) }\end{array}$ & $\lambda_{1}$ & $\alpha_{10}$ & $\alpha_{11}$ & $\alpha_{12}$ & $\lambda_{2}$ & $\alpha_{20}$ & $\alpha_{21}$ & $\alpha_{22}$ \\
\hline \multirow{6}{*}{$(30,10,15)$} & \multirow{2}{*}{1} & MLE & $\begin{array}{c}0.3686 \\
(0.0954)\end{array}$ & $\begin{array}{c}0.7009 \\
(0.1027)\end{array}$ & $\begin{array}{c}0.5129 \\
(0.1015)\end{array}$ & $\begin{array}{c}0.8813 \\
(0.1257)\end{array}$ & $\begin{array}{c}0.2615 \\
(0.0864)\end{array}$ & $\begin{array}{c}0.5946 \\
(0.1074)\end{array}$ & $\begin{array}{c}0.4346 \\
(0.0953)\end{array}$ & $\begin{array}{c}0.6516 \\
(0.1078)\end{array}$ \\
\hline & & Bayes & $\begin{array}{c}0.3488 \\
(0.0818)\end{array}$ & $\begin{array}{c}0.6897 \\
(0.0985)\end{array}$ & $\begin{array}{c}0.4913 \\
(0.0916)\end{array}$ & $\begin{array}{c}0.627 \\
(0.1051)\end{array}$ & $\begin{array}{c}0.2472 \\
(0.0749)\end{array}$ & $\begin{array}{c}0.5751 \\
(0.0905)\end{array}$ & $\begin{array}{c}0.4053 \\
(0.0895)\end{array}$ & $\begin{array}{c}0.6218 \\
(0.0971)\end{array}$ \\
\hline & \multirow[b]{2}{*}{2} & MLE & $\begin{array}{c}0.3617 \\
(0.0946)\end{array}$ & $\begin{array}{c}0.7011 \\
(0.1033)\end{array}$ & $\begin{array}{c}0.5124 \\
(0.0977)\end{array}$ & $\begin{array}{c}0.8816 \\
(0.1245)\end{array}$ & $\begin{array}{c}0.2646 \\
(0.0872)\end{array}$ & $\begin{array}{c}0.5975 \\
(0.1077)\end{array}$ & $\begin{array}{c}0.4334 \\
(0.0973)\end{array}$ & $\begin{array}{c}0.6469 \\
(0.1032)\end{array}$ \\
\hline & & Bayes & $\begin{array}{c}0.3472 \\
(0.0812)\end{array}$ & $\begin{array}{c}0.6814 \\
(0.0984)\end{array}$ & $\begin{array}{c}0.4923 \\
(0.0917)\end{array}$ & $\begin{array}{c}0.6262 \\
(0.1049)\end{array}$ & $\begin{array}{c}0.2496 \\
(0.0775)\end{array}$ & $\begin{array}{c}0.5742 \\
(0.9118)\end{array}$ & $\begin{array}{c}0.4054 \\
(0.0898)\end{array}$ & $\begin{array}{c}0.6205 \\
(0.0968)\end{array}$ \\
\hline & \multirow{2}{*}{3} & MLE & $\begin{array}{c}0.3662 \\
(0.0996)\end{array}$ & $\begin{array}{c}0.6977 \\
(0.1034)\end{array}$ & $\begin{array}{c}0.5117 \\
(0.0966)\end{array}$ & $\begin{array}{c}0.8792 \\
(0.1247)\end{array}$ & $\begin{array}{c}0.2668 \\
(0.0899)\end{array}$ & $\begin{array}{c}0.5984 \\
(0.1073)\end{array}$ & $\begin{array}{c}0.4346 \\
(0.0959)\end{array}$ & $\begin{array}{c}0.6493 \\
(0.1013)\end{array}$ \\
\hline & & Bayes & $\begin{array}{c}0.3439 \\
(0.0811)\end{array}$ & $\begin{array}{c}0.6798 \\
(0.0981)\end{array}$ & $\begin{array}{c}0.4933 \\
(0.0914)\end{array}$ & $\begin{array}{c}0.8524 \\
(0.1056)\end{array}$ & $\begin{array}{c}0.2493 \\
(0.0765)\end{array}$ & $\begin{array}{c}0.5741 \\
(0.0969)\end{array}$ & $\begin{array}{c}0.4051 \\
(0.0899)\end{array}$ & $\begin{array}{c}0.6218 \\
(0.0964)\end{array}$ \\
\hline \multirow{6}{*}{$(30,15,20)$} & \multirow[b]{2}{*}{1} & MLE & $\begin{array}{c}0.3482 \\
(0.0877)\end{array}$ & $\begin{array}{c}0.6854 \\
(0.0982)\end{array}$ & $\begin{array}{c}0.4979 \\
(0.0923)\end{array}$ & $\begin{array}{c}0.8698 \\
(0.1155)\end{array}$ & $\begin{array}{c}0.2567 \\
(0.0759)\end{array}$ & $\begin{array}{c}0.5854 \\
(0.0981)\end{array}$ & $\begin{array}{c}0.4209 \\
(0.0877)\end{array}$ & $\begin{array}{c}0.6385 \\
(0.0971)\end{array}$ \\
\hline & & Bayes & $\begin{array}{c}0.3341 \\
(0.0775)\end{array}$ & $\begin{array}{c}0.6774 \\
(0.0902)\end{array}$ & $\begin{array}{c}0.483 \\
(0.0876)\end{array}$ & $\begin{array}{c}0.844 \\
(0.0989)\end{array}$ & $\begin{array}{c}0.2457 \\
(0.0664)\end{array}$ & $\begin{array}{c}0.5695 \\
(0.0821)\end{array}$ & $\begin{array}{c}0.3962 \\
(0.0812)\end{array}$ & $\begin{array}{c}0.6149 \\
(0.0886)\end{array}$ \\
\hline & \multirow{2}{*}{2} & MLE & $\begin{array}{c}0.3476 \\
(0.0873)\end{array}$ & $\begin{array}{l}0.6871 \\
(0.984)\end{array}$ & $\begin{array}{c}0.4939 \\
(0.0926)\end{array}$ & $\begin{array}{c}0.8679 \\
(0.1157)\end{array}$ & $\begin{array}{c}0.2532 \\
(0.0782)\end{array}$ & $\begin{array}{c}0.5845 \\
(0.0981)\end{array}$ & $\begin{array}{c}0.4206 \\
(0.0876)\end{array}$ & $\begin{array}{c}0.6395 \\
(0.0978)\end{array}$ \\
\hline & & Bayes & $\begin{array}{c}0.3317 \\
(0.0772)\end{array}$ & $\begin{array}{c}0.6715 \\
(0.0903)\end{array}$ & $\begin{array}{c}0.4819 \\
(0.0875)\end{array}$ & $\begin{array}{c}0.8475 \\
(0.0985)\end{array}$ & $\begin{array}{c}0.2439 \\
(0.0667)\end{array}$ & $\begin{array}{c}0.5642 \\
(0.0822)\end{array}$ & $\begin{array}{c}0.3959 \\
(0.0819)\end{array}$ & $\begin{array}{c}0.6139 \\
(0.0883)\end{array}$ \\
\hline & \multirow{2}{*}{3} & MLE & $\begin{array}{c}0.3466 \\
(0.0875)\end{array}$ & $\begin{array}{c}0.6853 \\
(0.0989)\end{array}$ & $\begin{array}{c}0.4958 \\
(0.0924)\end{array}$ & $\begin{array}{c}0.8688 \\
(0.1163)\end{array}$ & $\begin{array}{c}0.2549 \\
(0.0784)\end{array}$ & $\begin{array}{c}0.5835 \\
(0.0987)\end{array}$ & $\begin{array}{c}0.4208 \\
(0.0874)\end{array}$ & $\begin{array}{c}0.6393 \\
(0.0973)\end{array}$ \\
\hline & & Bayes & $\begin{array}{c}0.3325 \\
(0.0778)\end{array}$ & $\begin{array}{c}0.6703 \\
(0.0901)\end{array}$ & $\begin{array}{c}0.4769 \\
(0.0867)\end{array}$ & $\begin{array}{c}0.8395 \\
(0.0987)\end{array}$ & $\begin{array}{c}0.2427 \\
(0.0667)\end{array}$ & $\begin{array}{c}0.5624 \\
(0.0821)\end{array}$ & $\begin{array}{c}0.3955 \\
(0.0818)\end{array}$ & $\begin{array}{c}0.6129 \\
(0.0886)\end{array}$ \\
\hline
\end{tabular}


TABle 3: Continued.

\begin{tabular}{|c|c|c|c|c|c|c|c|c|c|c|}
\hline$\left(n, m_{1}, m_{2}\right)$ & Scheme & $\begin{array}{c}\text { Estimator } \\
\text { (MSE) }\end{array}$ & $\lambda_{1}$ & $\alpha_{10}$ & $\alpha_{11}$ & $\alpha_{12}$ & $\lambda_{2}$ & $\alpha_{20}$ & $\alpha_{21}$ & $\alpha_{22}$ \\
\hline \multirow{9}{*}{$(40,15,20)$} & \multirow[b]{2}{*}{1} & MLE & $\begin{array}{c}0.3355 \\
(0.0812)\end{array}$ & $\begin{array}{c}0.679 \\
(0.0924)\end{array}$ & $\begin{array}{c}0.4864 \\
(0.0856)\end{array}$ & $\begin{array}{c}0.8526 \\
(0.1021)\end{array}$ & $\begin{array}{c}0.2508 \\
(0.0719)\end{array}$ & $\begin{array}{c}0.5712 \\
(0.0887)\end{array}$ & $\begin{array}{c}0.4083 \\
(0.0815)\end{array}$ & $\begin{array}{c}0.6296 \\
(0.0876)\end{array}$ \\
\hline & & Bayes & $\begin{array}{c}0.3255 \\
(0.0702)\end{array}$ & $\begin{array}{c}0.6671 \\
(0.0833)\end{array}$ & $\begin{array}{c}0.4681 \\
(0.0756)\end{array}$ & $\begin{array}{c}0.8358 \\
(0.0914)\end{array}$ & $\begin{array}{c}0.2417 \\
(0.0603)\end{array}$ & $\begin{array}{c}0.5546 \\
(0.0767)\end{array}$ & $\begin{array}{c}0.386 \\
(0.0744)\end{array}$ & $\begin{array}{c}0.6025 \\
(0.0807)\end{array}$ \\
\hline & \multirow{3}{*}{2} & MLE & $\begin{array}{c}0.3358 \\
(0.0814)\end{array}$ & $\begin{array}{c}0.6755 \\
(0.0914)\end{array}$ & $\begin{array}{c}0.4822 \\
(0.0851)\end{array}$ & $\begin{array}{c}0.8588 \\
(0.1026)\end{array}$ & $\begin{array}{c}0.2445 \\
(0.0718)\end{array}$ & $\begin{array}{c}0.573 \\
(0.0878)\end{array}$ & $\begin{array}{c}0.4077 \\
(0.0814)\end{array}$ & $\begin{array}{c}0.6305 \\
(0.0881)\end{array}$ \\
\hline & & \multirow{2}{*}{ Bayes } & 0.3254 & 0.6644 & 0.4669 & 0.8354 & 0.2312 & 0.5539 & 0.3856 & 0.6022 \\
\hline & & & $(0.0706)$ & $(0.0839)$ & $(0.0756)$ & $(0.0914)$ & $(0.0605)$ & $(0.0766)$ & $(0.0748)$ & $(0.0809)$ \\
\hline & \multirow{4}{*}{3} & \multirow{2}{*}{ MLE } & 0.3368 & 0.6768 & 0.4865 & 0.8577 & 0.2448 & 0.5721 & 0.4091 & 0.6317 \\
\hline & & & $(0.0818)$ & $(0.0915)$ & $(0.0855)$ & $(0.1027)$ & $(0.0713)$ & $(0.0873)$ & $(0.0812)$ & $(0.0891)$ \\
\hline & & \multirow{2}{*}{ Bayes } & 0.3256 & 0.668 & 0.4686 & 0.833 & 0.2348 & 0.5537 & 0.3879 & 0.6027 \\
\hline & & & $(0.0708)$ & $(0.0837)$ & $(0.0758)$ & $(0.0911)$ & $(0.0607)$ & $(0.0763)$ & $(0.0748)$ & $(0.0811)$ \\
\hline \multirow{12}{*}{$(40,20,25)$} & \multirow{4}{*}{1} & \multirow{2}{*}{ MLE } & 0.3229 & 0.6655 & 0.4721 & 0.6633 & 0.246 & 0.4029 & 0.2338 & 0.471 \\
\hline & & & $(0.0748)$ & $(0.0836)$ & $(0.0768)$ & $(0.0941)$ & $(0.0661)$ & $(0.0803)$ & $(0.0777)$ & $(0.0802)$ \\
\hline & & \multirow{2}{*}{ Bayes } & 0.3123 & 0.6523 & 0.4579 & 0.8257 & 0.2335 & 0.5483 & 0.3727 & 0.6018 \\
\hline & & & $(0.0644)$ & $(0.0765)$ & $(0.0675)$ & $(0.0885)$ & $(0.0579)$ & $(0.0696)$ & $(0.0705)$ & $(0.0731)$ \\
\hline & \multirow{4}{*}{2} & \multirow{2}{*}{ MLE } & 0.3213 & 0.6656 & 0.473 & 0.8479 & 0.2365 & 0.5621 & 0.3973 & 0.6262 \\
\hline & & & $(0.0746)$ & $(0.0833)$ & $(0.0766)$ & $(0.0949)$ & $(0.0669)$ & $(0.0801)$ & $(0.0772)$ & $(0.0808)$ \\
\hline & & \multirow{2}{*}{ Bayes } & 0.3127 & 0.6502 & 0.456 & 0.8259 & 0.2247 & 0.5489 & 0.3737 & 0.6019 \\
\hline & & & $(0.0642)$ & $(0.0762)$ & $(0.0674)$ & $(0.0888)$ & $(0.0577)$ & $(0.0697)$ & $(0.0701)$ & $(0.0729)$ \\
\hline & \multirow{4}{*}{3} & \multirow{2}{*}{ MLE } & 0.3215 & 0.6625 & 0.4767 & 0.6692 & 0.2361 & 0.5628 & 0.2324 & 0.6212 \\
\hline & & & $(0.0746)$ & $(0.0839)$ & $(0.0767)$ & $(0.0952)$ & $(0.0662)$ & $(0.0809)$ & $(0.0774)$ & $(0.0801)$ \\
\hline & & \multirow{2}{*}{ Bayes } & 0.3141 & 0.651 & 0.4563 & 0.8232 & 0.2241 & 0.5479 & 0.3727 & 0.5931 \\
\hline & & & $(0.0641)$ & $(0.0761)$ & $(0.0679)$ & $(0.0887)$ & $(0.0573)$ & $(0.0699)$ & $(0.0703)$ & $(0.0731)$ \\
\hline \multirow{12}{*}{$(50,20,25)$} & & MLE & 0.3016 & 0.6596 & 0.4641 & 0.8265 & 0.2343 & 0.5522 & 0.3852 & 0.6114 \\
\hline & 1 & & $(0.0621)$ & $(0.0751)$ & $(0.0656)$ & $(0.0852)$ & $(0.0576)$ & $(0.0709)$ & $(0.0675)$ & $(0.0707)$ \\
\hline & 1 & & 0.2924 & 0.6395 & 0.4429 & 0.8187 & 0.2271 & 0.5319 & 0.364 & 0.5807 \\
\hline & & Bayes & $(0.0549)$ & $(0.0674)$ & $(0.0567)$ & $(0.0779)$ & $(0.0509)$ & $(0.0623)$ & $(0.0605)$ & $(0.0629)$ \\
\hline & & MLE & 0.3017 & 0.6587 & 0.3418 & 0.8281 & 0.2248 & 0.5515 & 0.3879 & 0.6114 \\
\hline & 2 & & $(0.0627)$ & $(0.0748)$ & $(0.0657)$ & $(0.0851)$ & $(0.0582)$ & $(0.0706)$ & $(0.0674)$ & $(0.0711)$ \\
\hline & 2 & & 0.2916 & 0.6387 & 0.4457 & 0.8152 & 0.2174 & 0.5329 & 0.3644 & 0.5832 \\
\hline & & Bayes & $(0.0549)$ & $(0.0679)$ & $(0.0562)$ & $(0.0778)$ & $(0.0505)$ & $(0.0619)$ & $(0.0607)$ & $(0.0621)$ \\
\hline & & MLE & 0.3093 & 0.6537 & 0.4651 & 0.8274 & 0.2269 & 0.5518 & 0.3852 & 0.6103 \\
\hline & 3 & & $(0.0626)$ & $(0.0749)$ & $(0.0655)$ & $(0.0859)$ & $(0.0577)$ & $(0.0701)$ & $(0.0679)$ & $(0.0712)$ \\
\hline & & & 0.297 & 0.6359 & 0.4455 & 0.8176 & 0.2169 & 0.5325 & 0.3628 & 0.5846 \\
\hline & & Bayes & $(0.0544)$ & $(0.0677)$ & $(0.0564)$ & $(0.0781)$ & $(0.0507)$ & $(0.0621)$ & $(0.0603)$ & $(0.0624)$ \\
\hline & & MLE & 0.2828 & 0.6414 & 0.4527 & 0.8164 & 0.2251 & 0.5362 & 0.3724 & 0.6059 \\
\hline & 1 & & $(0.0508)$ & $(0.0639)$ & $(0.0511)$ & $(0.0761)$ & $(0.0471)$ & $(0.0607)$ & $(0.0511)$ & $(0.0681)$ \\
\hline & 1 & & 0.2752 & 0.6345 & 0.4318 & 0.8033 & 0.2169 & 0.4379 & 0.3552 & 0.5742 \\
\hline & & Bayes & $(0.0407)$ & $(0.0563)$ & $(0.0507)$ & $(0.0688)$ & $(0.0404)$ & $(0.0508)$ & $(0.0479)$ & $(0.0524)$ \\
\hline & & & 0.2892 & 0.6423 & 0.4517 & 0.8151 & 0.2163 & 0.5391 & 0.3747 & 0.4871 \\
\hline & 2 & MLE & $(0.0509)$ & $(0.0637)$ & $(0.0507)$ & $(0.0768)$ & $(0.0478)$ & $(0.0611)$ & $(0.0518)$ & $(0.0678)$ \\
\hline$(50$ & 2 & & 0.273 & 0.6233 & 0.4312 & 0.8016 & 0.2046 & 0.5216 & 0.3585 & 0.5762 \\
\hline & & Bayes & $(0.0403)$ & $(0.0561)$ & $(0.0509)$ & $(0.0685)$ & $(0.0405)$ & $(0.0509)$ & $(0.0482)$ & $(0.0531)$ \\
\hline & & & 0.2851 & 0.6443 & 0.4508 & 0.815 & 0.2152 & 0.5377 & 0.3755 & 0.6072 \\
\hline & 3 & MLE & $(0.0509)$ & $(0.0635)$ & $(0.0505)$ & $(0.0769)$ & $(0.0477)$ & $(0.0609)$ & $(0.0517)$ & $(0.0682)$ \\
\hline & 3 & Bayes & 0.2716 & 0.6276 & 0.4352 & 0.803 & 0.2062 & 0.5224 & 0.3595 & 0.5766 \\
\hline & & Dayes & $(0.0406)$ & $(0.0558)$ & $(0.0506)$ & $(0.0687)$ & $(0.0407)$ & $(0.0507)$ & $(0.0481)$ & $(0.0527)$ \\
\hline
\end{tabular}

From Table 7, we can obtain that there is little difference between the MLEs and BEs of the unknown parameters. The lengths for the HPD CIs are shorter than the ACIs in most cases. For the given significance value, the accuracy of the HPD CIs is better than the ACIs.

Here, aiming at the above data analysis, there exists a question on how good the proposed MOBG model fits the data. That is, there have been multiple goodness-of-fit testing methods for univariate distributions. However, for bivariate distribution, the goodness-of-fit testing methods are less.
Thus, to verify the above fit results of data and distribution, we mainly used the method which is from the Kundu and Gupta [27], and we verified two margins and the minimum lifetime by using the Kolmogorov-Smirnov test (K-S test).

Give the significance value $\gamma=5 \%$, the K-S statistic and the corresponding $p$ values under different failure numbers are obtained, and the result is presented in Table 8 . The comparison charts for empirical distribution and its fitted distribution of the sample under each stress are shown in Figures 1-3. 
TABle 4: The CPs and AILs of the confidence intervals for unknown parameters $(\tau=0.5)$.

\begin{tabular}{|c|c|c|c|c|c|c|c|c|c|c|}
\hline$\left(n, m_{1}, m_{2}\right)$ & Scheme & $\begin{array}{c}\mathrm{CP} \\
(\mathrm{AIL})\end{array}$ & $\lambda_{1}$ & $\alpha_{10}$ & $\alpha_{11}$ & $\alpha_{12}$ & $\lambda_{2}$ & $\alpha_{20}$ & $\alpha_{21}$ & $\alpha_{22}$ \\
\hline \multirow{12}{*}{$(30,10,15)$} & \multirow{4}{*}{1} & \multirow{2}{*}{ MLE } & 84.7 & 84.7 & 84.4 & 85.1 & 85.2 & 84.6 & 84.7 & 85.8 \\
\hline & & & $(0.2892)$ & $(0.3312)$ & $(0.2763)$ & $(0.3762)$ & $(0.2195)$ & $(0.2953)$ & $(0.2682)$ & $(0.3152)$ \\
\hline & & \multirow{2}{*}{ Bayes } & 88.1 & 87.1 & 88.1 & 88.7 & 89.1 & 90.2 & 88.4 & 89.3 \\
\hline & & & $(0.2768)$ & $(0.3198)$ & $(0.2684)$ & $(0.3638)$ & $(0.2079)$ & $(0.2861)$ & $(0.2586)$ & $(0.3055)$ \\
\hline & \multirow{4}{*}{2} & \multirow{2}{*}{ MLE } & 84.3 & 84.5 & 85.9 & 85.2 & 85.1 & 84.2 & 84.5 & 85.3 \\
\hline & & & $(0.2868)$ & $(0.3237)$ & $(0.2748)$ & $(0.3724)$ & $(0.2174)$ & $(0.2972)$ & $(0.2684)$ & $(0.3154)$ \\
\hline & & \multirow{2}{*}{ Bayes } & 87.9 & 88.2 & 89.2 & 88.7 & 89.5 & 90.1 & 88.2 & 89.1 \\
\hline & & & $(0.2732)$ & $(0.3158)$ & $(0.2681)$ & $(0.3628)$ & $(0.2738)$ & $(0.2822)$ & $(0.2558)$ & $(0.3037)$ \\
\hline & \multirow{4}{*}{3} & MLE & 84.2 & 84.1 & 85.1 & $85(0.3713)$ & 85.2 & 84.9 & 84.4 & 85.3 \\
\hline & & & $(0.2872)$ & $(0.3254)$ & $(0.2775)$ & (נסוסיט(ס) & $(0.2174)$ & $(0.2961)$ & $(0.2653)$ & $(0.3171)$ \\
\hline & & Baves & 87.2 & 88.1 & 89.1 & 88.1 & 89.4 & 89.9 & 88.2 & 89.2 \\
\hline & & Dayes & $(0.2752)$ & $(0.3149)$ & $(0.2669)$ & $(0.3652)$ & $(0.2044)$ & $(0.2846)$ & $(0.2393)$ & $(0.3024)$ \\
\hline \multirow{11}{*}{$(30,15,20)$} & \multirow{3}{*}{1} & MLE & $86(0.2675)$ & $\begin{array}{c}86.3 \\
(0.3084)\end{array}$ & $\begin{array}{c}85.2 \\
(0.2569)\end{array}$ & $\begin{array}{c}86.5 \\
(0.3546)\end{array}$ & $87(0.1989)$ & $\begin{array}{c}85.2 \\
(0.2773)\end{array}$ & $\begin{array}{c}86.8 \\
(0.2489)\end{array}$ & $87(0.2965)$ \\
\hline & & Bayes & 90.3 & 89.2 & 89.6 & 89.2 & 90.3 & 91.1 & 89.9 & 91.1 \\
\hline & & Dayes & $(0.2546)$ & $(0.2969)$ & $(0.2478)$ & $(0.3412)$ & $(0.1891)$ & $(0.2684)$ & $(0.2372)$ & $(0.2858)$ \\
\hline & \multirow{4}{*}{2} & MIF & 85.6 & 85.7 & 86.3 & 87.2 & 86.7 & 86.2 & 85.8 & 87.6 \\
\hline & & MLE & $(0.2672)$ & $(0.3058)$ & $(0.2545)$ & $(0.3553)$ & $(0.1987)$ & $(0.2785)$ & $(0.2487)$ & $(0.2966)$ \\
\hline & & & 89.6 & 90.3 & 89.4 & 89.3 & 90.2 & 91.8 & 89.2 & 90.2 \\
\hline & & Bayes & $(0.2549)$ & $(0.2964)$ & $(0.2469)$ & $(0.3454)$ & $(0.1871)$ & $(0.2684)$ & $(0.2379)$ & $(0.2845)$ \\
\hline & \multirow{4}{*}{3} & MLE & 85.4 & 85.6 & 86.4 & $86(0.3591)$ & $87(0.2025)$ & 86.2 & 85.6 & 86.8 \\
\hline & & & $(0.2689)$ & $(0.3074)$ & $(0.2582)$ & & & $(0.2778)$ & $(0.2483)$ & $(0.2954)$ \\
\hline & & Bayes & 89.1 & 89.7 & 90.1 & 89.3 & 90.4 & 90.1 & 89.3 & 90.2 \\
\hline & & Dayes & $(0.2571)$ & $(0.2956)$ & $(0.2448)$ & $(0.3447)$ & $(0.1887)$ & $(0.2676)$ & $(0.2368)$ & $(0.2822)$ \\
\hline \multirow{10}{*}{$(40,15,20)$} & \multirow{3}{*}{1} & MLE & $\begin{array}{c}87.4 \\
(0.2527)\end{array}$ & $\begin{array}{c}87.7 \\
(0.2873)\end{array}$ & $\begin{array}{c}86.1 \\
(0.2366)\end{array}$ & $\begin{array}{c}86.8 \\
(0.3362)\end{array}$ & $88(0.1831)$ & $\begin{array}{c}87.3 \\
(0.2569)\end{array}$ & $88(0.2287)$ & $89(0.2778)$ \\
\hline & & Baves & 91.4 & 92.1 & 92.9 & 91.1 & 91.6 & 92.6 & $92(02185)$ & 92.3 \\
\hline & & Dayes & $(0.2376)$ & $(0.2765)$ & $(0.2264)$ & $(0.3254)$ & $(0.1683)$ & $(0.2488)$ & (0.210J) & $(0.2687)$ \\
\hline & \multirow{3}{*}{2} & MLE & $87(0.2545)$ & 86.6 & 88.4 & 88.5 & 88.3 & 88.8 & 87.4 & 89.4 \\
\hline & & & & $(0.2853)$ & $(0.2347)$ & $(0.3376)$ & $(0.1826)$ & $(0.2595)$ & $(0.2273)$ & $(0.2795)$ \\
\hline & & Bayes & $\begin{array}{c}91.5 \\
(02423)\end{array}$ & $\begin{array}{c}92.3 \\
(02733)\end{array}$ & 91.6 & $\begin{array}{c}91.6 \\
(03262)\end{array}$ & 91.6 & 92.2 & $\begin{array}{c}91.5 \\
(02177)\end{array}$ & 92.8 \\
\hline & \multirow{4}{*}{3} & & 87.1 & 86.9 & 87.9 & 88.1 & & 88.1 & $\begin{array}{c}(0.21 / 1) \\
87.2\end{array}$ & $\begin{array}{c}(0.2693) \\
88.7\end{array}$ \\
\hline & & MLE & $(0.2579)$ & $(0.2893)$ & $(0.2376)$ & $(0.3368)$ & $88(0.1852)$ & $(0.2548)$ & $(0.2297)$ & $(0.2762)$ \\
\hline & & Bayes & 91.1 & $92(0.2758)$ & 91.2 & 91.1 & 91.5 & 92.2 & 91.3 & 92.3 \\
\hline & & Dayes & $(0.2425)$ & & $(0.2269)$ & $(0.3245)$ & $(0.1685)$ & $(0.2481)$ & $(0.2183)$ & $(0.2679)$ \\
\hline \multirow{10}{*}{$(40,20,25)$} & 1 & MLE & $89(0.2333)$ & $89(0.2736)$ & $88(0.2149)$ & $\begin{array}{c}88.3 \\
(0.3258)\end{array}$ & $90(0.1684)$ & $\begin{array}{c}89.6 \\
(0.2335)\end{array}$ & $90(0.2142)$ & $\begin{array}{c}90.2 \\
(0.2611)\end{array}$ \\
\hline & 1 & Bayes & 94.2 & 94.1 & 94.5 & 93.5 & 94.1 & 94.2 & 93.3 & 93.2 \\
\hline & & Bayes & $(0.2273)$ & $(0.2543)$ & $(0.2076)$ & $(0.3062)$ & $(0.1482)$ & $(0.2246)$ & $(0.1964)$ & $(0.2563)$ \\
\hline & 2 & MLE & $\begin{array}{c}89.5 \\
(0.2362)\end{array}$ & $89(0.2698)$ & $\begin{array}{c}89.5 \\
(0.2182)\end{array}$ & $\begin{array}{c}89.5 \\
(0.3221)\end{array}$ & $\begin{array}{c}89.2 \\
(0.1651)\end{array}$ & $\begin{array}{c}89.8 \\
(0.2361)\end{array}$ & $89(0.2138)$ & $91(0.2578)$ \\
\hline & 2 & Baves & 94.4 & 94.9 & 94.8 & 94.3 & 93.9 & 94.8 & 93.5 & 94.4 \\
\hline & & Bayes & $(0.2256)$ & $(0.2577)$ & $(0.2086)$ & $(0.3076)$ & $(0.1463)$ & $(0.2265)$ & $(0.1967)$ & $(0.2467)$ \\
\hline & & MLE & 89.3 & 88.2 & 89.2 & 89.3 & 89.7 & 89.7 & 88.7 & 89.9 \\
\hline & 3 & MLF & $(0.2344)$ & $(0.2756)$ & $(0.2226)$ & $(0.3221)$ & $(0.1692)$ & $(0.2368)$ & $(0.2139)$ & $(0.2572)$ \\
\hline & 3 & Bayes & 94.3 & 94.8 & $94(0.2077)$ & 94.3 & 93.3 & 94.1 & 93.3 & 94.1 \\
\hline & & & $(0.2272)$ & $(0.2566)$ & & $(0.3089)$ & $(0.1469)$ & $(0.2344)$ & $(0.1987)$ & $(0.2452)$ \\
\hline & & MLE & $\begin{array}{c}90.9 \\
(0.2166)\end{array}$ & $\begin{array}{c}89.9 \\
(0.2547)\end{array}$ & $\begin{array}{c}89.9 \\
(0.2051)\end{array}$ & $\begin{array}{c}89.5 \\
(0.3089)\end{array}$ & $\begin{array}{c}90.8 \\
(0.1474)\end{array}$ & $\begin{array}{c}90.8 \\
(0.2148)\end{array}$ & $\begin{array}{c}91.8 \\
(0.1989)\end{array}$ & $91.3(0.248)$ \\
\hline & 1 & & 95.7 & 96.1 & 95.6 & & 96.8 & 95.9 & 95.2 & 95.1 \\
\hline & & Bayes & $(0.2066)$ & $(0.2342)$ & $(0.1878)$ & $95(0.2854)$ & $(0.1324)$ & $(0.2078)$ & $(0.1732)$ & $(0.2283)$ \\
\hline & & MIF & 90.8 & 89.2 & 90.7 & 90.2 & 90.9 & 90.1 & 90.8 & 91.7 \\
\hline$(50,20,25)$ & 2 & & $(0.2185)$ & $(0.2532)$ & $(0.2025)$ & $(0.3097)$ & $(0.1442)$ & $(0.2176)$ & $(0.1942)$ & $(0.2423)$ \\
\hline$(50,20,25)$ & 2 & & 95.2 & 96.2 & 95.8 & 97.8 & 96.2 & 96.7 & 97.8 & 96.4 \\
\hline & & Bayes & $(0.2068)$ & $(0.2338)$ & $(0.1853)$ & $(0.2887)$ & $(0.1292)$ & $(0.2083)$ & $(0.1784)$ & $(0.2256)$ \\
\hline & & MIF & 90.5 & 89.1 & 90.2 & 90.1 & 90.3 & 90.1 & 90.1 & 91.1 \\
\hline & 3 & NILE & $(0.2169)$ & $(0.2541)$ & $(0.2015)$ & $(0.3076)$ & $(0.1458)$ & $(0.2234)$ & $(0.1935)$ & $(0.2373)$ \\
\hline & 3 & Bayes & $\begin{array}{c}95.1 \\
(0.2089)\end{array}$ & $\begin{array}{c}96.1 \\
(0.2429)\end{array}$ & $\begin{array}{c}95.2 \\
(0.1871)\end{array}$ & $97(0.2895)$ & $\begin{array}{c}96.4 \\
(0.1282)\end{array}$ & $\begin{array}{c}96.3 \\
(0.2143)\end{array}$ & $\begin{array}{c}96.8 \\
(0.1763)\end{array}$ & $\begin{array}{c}96.3 \\
(0.2245)\end{array}$ \\
\hline
\end{tabular}


TABle 4: Continued.

\begin{tabular}{|c|c|c|c|c|c|c|c|c|c|c|}
\hline$\left(n, m_{1}, m_{2}\right)$ & Scheme & $\begin{array}{c}\mathrm{CP} \\
(\mathrm{AIL})\end{array}$ & $\lambda_{1}$ & $\alpha_{10}$ & $\alpha_{11}$ & $\alpha_{12}$ & $\lambda_{2}$ & $\alpha_{20}$ & $\alpha_{21}$ & $\alpha_{22}$ \\
\hline \multirow{6}{*}{$(50,25,30)$} & \multirow{2}{*}{1} & MLE & $\begin{array}{c}92.7 \\
(0.1967)\end{array}$ & $\begin{array}{c}91.7 \\
(0.2357)\end{array}$ & $\begin{array}{c}91.6 \\
(0.1879)\end{array}$ & $\begin{array}{c}92.1 \\
(0.2865)\end{array}$ & $\begin{array}{c}91.5 \\
(0.1263)\end{array}$ & $\begin{array}{c}91.6 \\
(0.1971)\end{array}$ & $\begin{array}{c}92.4 \\
(0.1784)\end{array}$ & $93(0.2266)$ \\
\hline & & Bayes & $\begin{array}{c}97.1 \\
(0.1866)\end{array}$ & $\begin{array}{c}97.8 \\
(0.2151)\end{array}$ & $\begin{array}{c}97.7 \\
(0.1657)\end{array}$ & $\begin{array}{c}97.9 \\
(0.2648)\end{array}$ & $\begin{array}{c}98.3 \\
(0.1132)\end{array}$ & $\begin{array}{c}97.9 \\
(0.1843)\end{array}$ & $\begin{array}{c}98.4 \\
(0.1579)\end{array}$ & $\begin{array}{c}97.8 \\
(0.2058)\end{array}$ \\
\hline & \multirow{2}{*}{2} & MLE & $\begin{array}{c}91.6 \\
(0.1971)\end{array}$ & $\begin{array}{c}91.4 \\
(0.2356)\end{array}$ & $\begin{array}{c}92.6 \\
(0.1857)\end{array}$ & $\begin{array}{c}92.2 \\
(0.2859)\end{array}$ & $\begin{array}{c}92.5 \\
(0.1287)\end{array}$ & $\begin{array}{c}92.4 \\
(0.1954)\end{array}$ & $\begin{array}{c}92.8 \\
(0.1784)\end{array}$ & $\begin{array}{c}93.5 \\
(0.2283)\end{array}$ \\
\hline & & Bayes & $\begin{array}{c}97.6 \\
(0.1832)\end{array}$ & $\begin{array}{c}97.3 \\
(0.2152)\end{array}$ & $\begin{array}{c}97.5 \\
(0.1669)\end{array}$ & $98(0.2666)$ & $\begin{array}{c}98.6 \\
(0.1187)\end{array}$ & $\begin{array}{c}97.5 \\
(0.1824)\end{array}$ & $\begin{array}{c}98.7 \\
(0.1576)\end{array}$ & $\begin{array}{c}97.8 \\
(0.2073)\end{array}$ \\
\hline & \multirow{2}{*}{3} & MLE & $\begin{array}{c}91.4 \\
(0.1978)\end{array}$ & $\begin{array}{c}91.2 \\
(0.2352)\end{array}$ & $\begin{array}{c}92.1 \\
(0.1834)\end{array}$ & $\begin{array}{c}92.2 \\
(0.2858)\end{array}$ & $\begin{array}{c}92.1 \\
(0.1266)\end{array}$ & $\begin{array}{c}92.2 \\
(0.2026)\end{array}$ & $\begin{array}{c}92.1 \\
(0.1728)\end{array}$ & $\begin{array}{c}93.2 \\
(0.2134)\end{array}$ \\
\hline & & Bayes & $\begin{array}{c}97.5 \\
(0.1852)\end{array}$ & $\begin{array}{c}97.1 \\
(0.2276)\end{array}$ & $\begin{array}{c}97.9 \\
(0.1653)\end{array}$ & $\begin{array}{c}98.1 \\
(0.2673)\end{array}$ & $\begin{array}{c}97.5 \\
(0.1142)\end{array}$ & $\begin{array}{c}97.1 \\
(0.1945)\end{array}$ & $98(0.1575)$ & $\begin{array}{c}97.8 \\
(0.2065)\end{array}$ \\
\hline
\end{tabular}

TABLE 5: The CPs and AILs of the confidence intervals for unknown parameters $(\tau=1)$.

\begin{tabular}{|c|c|c|c|c|c|c|c|c|c|c|}
\hline$\left(n, m_{1}, m_{2}\right)$ & Scheme & $\begin{array}{l}\mathrm{CP} \\
\text { (AIL) }\end{array}$ & $\lambda_{1}$ & $\alpha_{10}$ & $\alpha_{11}$ & $\alpha_{12}$ & $\lambda_{2}$ & $\alpha_{20}$ & $\alpha_{21}$ & $\alpha_{22}$ \\
\hline \multirow{10}{*}{$(30,10,15)$} & \multirow{4}{*}{1} & \multirow{2}{*}{ MLE } & 84.7 & 84.3 & 84.3 & 84.9 & 85.2 & 84.5 & 84.3 & 85.1 \\
\hline & & & $(0.2886)$ & $(0.3294)$ & $(0.2739)$ & $(0.3737)$ & $(0.2186)$ & $(0.2922)$ & $(0.2662)$ & $(0.3135)$ \\
\hline & & \multirow{2}{*}{ Bayes } & 88.1 & 87.3 & 87.9 & 88.4 & 88.9 & 90.2 & 88.4 & 89.1 \\
\hline & & & $(0.2737)$ & $(0.3177)$ & $(0.2675)$ & $(0.3619)$ & $(0.2056)$ & $(0.2832)$ & $(0.2563)$ & $(0.3027)$ \\
\hline & \multirow{3}{*}{2} & MLE & $\begin{array}{c}84.5 \\
(0.2875)\end{array}$ & $\begin{array}{c}84.2 \\
(0.3246)\end{array}$ & $\begin{array}{c}84.3 \\
(0.2723)\end{array}$ & $\begin{array}{c}84.6 \\
(0.3732)\end{array}$ & $\begin{array}{c}85.1 \\
(0.2168)\end{array}$ & $\begin{array}{c}84.2 \\
(0.2952)\end{array}$ & $84.1(0.269)$ & $\begin{array}{c}84.8 \\
(0.3172)\end{array}$ \\
\hline & & \multirow[b]{2}{*}{ Bayes } & 88.1 & 87.1 & 87.6 & 88.2 & 88.2 & 89.7 & 88.2 & 89.2 \\
\hline & & & $(0.2755)$ & $(0.3168)$ & $(0.2659)$ & $(0.3625)$ & $(0.2027)$ & $(0.2821)$ & $(0.2528)$ & $(0.3018)$ \\
\hline & \multirow{3}{*}{3} & MLE & $\begin{array}{c}84.3 \\
(0.2881)\end{array}$ & $\begin{array}{c}84.2 \\
(0.3239)\end{array}$ & $\begin{array}{c}84.2 \\
(0.2751)\end{array}$ & $\begin{array}{c}84.4 \\
(0.3702)\end{array}$ & 85 (0.2189) & $84.1(0.297)$ & $\begin{array}{c}84.2 \\
(0.2611)\end{array}$ & $\begin{array}{c}84.6 \\
(0.3138)\end{array}$ \\
\hline & & \multirow{2}{*}{ Bayes } & 88.1 & 87.3 & 87.4 & 88.1 & 88.1 & 89.5 & 88.3 & 89.1 \\
\hline & & & $(0.2748)$ & $(0.3127)$ & $(0.2666)$ & $(0.3622)$ & $(0.1989)$ & $(0.2812)$ & $(0.2532)$ & $(0.3015)$ \\
\hline \multirow{10}{*}{$(30,15,20)$} & \multirow{4}{*}{1} & \multirow{2}{*}{ MLE } & 85.7 & 86.2 & 85.8 & 86.2 & 86.5 & 86.2 & 85.3 & 86.9 \\
\hline & & & $(0.2694)$ & $(0.3076)$ & $(0.2577)$ & $(0.3544)$ & $(0.1999)$ & $(0.2785)$ & $(0.2478)$ & $(0.2948)$ \\
\hline & & \multirow{2}{*}{ Bayes } & 90.5 & 89.1 & 89.6 & 89.1 & 90.1 & 90.1 & 89.4 & 90.1 \\
\hline & & & $(0.2569)$ & $(0.2965)$ & $(0.2485)$ & $(0.3426)$ & $(0.1885)$ & $(0.2676)$ & $(0.2388)$ & $(0.2869)$ \\
\hline & \multirow{2}{*}{2} & MLE & $\begin{array}{c}85.5 \\
(0.2684)\end{array}$ & $\begin{array}{c}86.1 \\
(0.3065)\end{array}$ & $\begin{array}{c}85.7 \\
(0.2588)\end{array}$ & $\begin{array}{c}86.4 \\
(0.3589)\end{array}$ & $\begin{array}{c}86.4 \\
(0.1997)\end{array}$ & $\begin{array}{c}86.1 \\
(0.2797)\end{array}$ & $86(0.2481)$ & $\begin{array}{c}86.2 \\
(0.2987)\end{array}$ \\
\hline & & Bayes & 90.2 & 89.3 & 89.6 & \multirow{2}{*}{$89(0.3456)$} & $90(0.1864)$ & 90.1 & 89.3 & 90.1 \\
\hline & & \multirow[b]{2}{*}{ MLE } & $\begin{array}{c}(0.2596) \\
85.4\end{array}$ & $\begin{array}{c}(0.2974) \\
86.1\end{array}$ & $\begin{array}{c}(0.2486) \\
85.7\end{array}$ & & 89.3 & $\begin{array}{c}(0.2679) \\
86.2\end{array}$ & $\begin{array}{c}(0.2356) \\
85.8\end{array}$ & $\begin{array}{c}(0.2852) \\
86.2\end{array}$ \\
\hline & \multirow{3}{*}{3} & & $(0.2702)$ & $(0.3096)$ & $(0.2571)$ & $(0.3605)$ & $(0.2041)$ & $(0.2798)$ & $(0.2495)$ & $(0.2962)$ \\
\hline & & \multirow{2}{*}{ Bayes } & \multirow{2}{*}{$90(0.2583)$} & 89.2 & 89.3 & 88.8 & 89.9 & 90.1 & 89.2 & 90.2 \\
\hline & & & & $(0.2977)$ & $(0.2463)$ & $(0.3414)$ & $(0.1887)$ & $(0.2675)$ & $(0.2369)$ & $(0.283)$ \\
\hline \multirow{9}{*}{$(40,15,20)$} & \multirow{3}{*}{1} & MLE & $\begin{array}{c}87.2 \\
(0.2513)\end{array}$ & $\begin{array}{c}87.3 \\
(0.2888)\end{array}$ & $\begin{array}{c}86.7 \\
(0.2369)\end{array}$ & $\begin{array}{c}86.7 \\
(0.3377)\end{array}$ & $\begin{array}{c}87.9 \\
(0.1812)\end{array}$ & $\begin{array}{c}87.3 \\
(0.2589)\end{array}$ & $\begin{array}{c}87.6 \\
(0.2277)\end{array}$ & $\begin{array}{c}88.5 \\
(0.2765)\end{array}$ \\
\hline & & & 91.2 & 91.6 & 92.6 & & 91.4 & 92.4 & 91.9 & 92.2 \\
\hline & & Bayes & $(0.2426)$ & $(0.2713)$ & $(0.2296)$ & $91(0.3249)$ & $(0.1697)$ & $(0.2484)$ & $(0.2197)$ & $(0.2696)$ \\
\hline & & MLE & $\begin{array}{c}87.2 \\
(0.2537)\end{array}$ & $\begin{array}{c}87.2 \\
(0.2876)\end{array}$ & $\begin{array}{c}86.3 \\
(0.2318)\end{array}$ & $\begin{array}{c}87.8 \\
(0.3399)\end{array}$ & $\begin{array}{c}87.8 \\
(0.1807)\end{array}$ & $\begin{array}{c}87.2 \\
(0.2596)\end{array}$ & $\begin{array}{c}87.5 \\
(0.2298)\end{array}$ & $\begin{array}{c}88.2 \\
(0.2787)\end{array}$ \\
\hline & 2 & & 91.1 & 91.2 & 92.7 & & 91.2 & 92.4 & 91.7 & \\
\hline & & Bayes & $(0.2419)$ & $(0.2737)$ & $(0.2278)$ & $91(0.3232)$ & $(0.1687)$ & $(0.2437)$ & $(0.2189)$ & $92(0.2683)$ \\
\hline & & MLE & $87(0.2562)$ & $\begin{array}{c}87.1 \\
(02908)\end{array}$ & $\begin{array}{c}86.1 \\
(02396)\end{array}$ & $\begin{array}{c}87.7 \\
(03356)\end{array}$ & $\begin{array}{c}87.8 \\
(01874)\end{array}$ & 87.2 & 87.5 & $\begin{array}{c}88.2 \\
(02785)\end{array}$ \\
\hline & 3 & & & (0.2908) & $(0.2396)$ & (0.3356) & (0.1874) & $(0.2585)$ & $(0.2303)$ & $(0.2785)$ \\
\hline & . & Bayes & $\begin{array}{c}90.9 \\
(0.2412)\end{array}$ & $\begin{array}{c}91.2 \\
(0.2765)\end{array}$ & $\begin{array}{c}92.1 \\
(0.2257)\end{array}$ & $\begin{array}{c}90.8 \\
(0.3275)\end{array}$ & $\begin{array}{c}91.2 \\
(0.1667)\end{array}$ & $\begin{array}{c}92.2 \\
(0.2493)\end{array}$ & $\begin{array}{c}91.7 \\
(0.2141)\end{array}$ & $92(0.2625)$ \\
\hline
\end{tabular}


TABle 5: Continued.

\begin{tabular}{|c|c|c|c|c|c|c|c|c|c|c|}
\hline$\left(n, m_{1}, m_{2}\right)$ & Scheme & $\begin{array}{c}\mathrm{CP} \\
\text { (AIL) }\end{array}$ & $\lambda_{1}$ & $\alpha_{10}$ & $\alpha_{11}$ & $\alpha_{12}$ & $\lambda_{2}$ & $\alpha_{20}$ & $\alpha_{21}$ & $\alpha_{22}$ \\
\hline \multirow{6}{*}{$(40,20,25)$} & \multirow{2}{*}{1} & MLE & $\begin{array}{c}88.6 \\
(0.2346)\end{array}$ & $\begin{array}{c}88.9 \\
(0.2724)\end{array}$ & $\begin{array}{c}87.9 \\
(0.2184)\end{array}$ & $\begin{array}{c}88.2 \\
(0.3212)\end{array}$ & $\begin{array}{c}89.5 \\
(0.1686)\end{array}$ & $\begin{array}{c}89.4 \\
(0.2342)\end{array}$ & $\begin{array}{c}89.1 \\
(0.2139)\end{array}$ & $\begin{array}{c}90.1 \\
(0.2601)\end{array}$ \\
\hline & & Bayes & $\begin{array}{c}93.8 \\
(0.2265)\end{array}$ & $94(0.2584)$ & $\begin{array}{c}94.3 \\
(0.2093)\end{array}$ & $\begin{array}{c}93.2 \\
(0.3086)\end{array}$ & $\begin{array}{c}94.1 \\
(0.1499)\end{array}$ & $\begin{array}{c}94.1 \\
(0.2253)\end{array}$ & $\begin{array}{c}93.7 \\
(0.1997)\end{array}$ & $94(0.2579)$ \\
\hline & \multirow{2}{*}{2} & MLE & $\begin{array}{c}88.4 \\
(0.2369)\end{array}$ & $\begin{array}{c}88.7 \\
(0.2692)\end{array}$ & $\begin{array}{c}87.9 \\
(0.2193)\end{array}$ & $\begin{array}{c}88.3 \\
(0.3215)\end{array}$ & $\begin{array}{c}89.2 \\
(0.1677)\end{array}$ & $\begin{array}{c}89.3 \\
(0.2372)\end{array}$ & $\begin{array}{c}89.1 \\
(0.2104)\end{array}$ & $90(0.2597)$ \\
\hline & & Bayes & $\begin{array}{c}93.8 \\
(0.2273)\end{array}$ & $\begin{array}{c}93.9 \\
(0.2595)\end{array}$ & $\begin{array}{c}94.4 \\
(0.2091)\end{array}$ & $\begin{array}{c}93.1 \\
(0.3093)\end{array}$ & $\begin{array}{c}93.9 \\
(0.1476)\end{array}$ & $94(0.2295)$ & $\begin{array}{c}93.4 \\
(0.1999)\end{array}$ & $\begin{array}{c}93.8 \\
(0.2448)\end{array}$ \\
\hline & \multirow[t]{2}{*}{3} & MLE & $\begin{array}{c}88.4 \\
(0.235)\end{array}$ & $\begin{array}{c}88.7 \\
(0.2719)\end{array}$ & $\begin{array}{c}87.8 \\
(0.2219)\end{array}$ & $88(0.3241)$ & $\begin{array}{c}89.2 \\
(0.1685)\end{array}$ & $\begin{array}{c}89.3 \\
(0.2393)\end{array}$ & $89(0.2123)$ & $\begin{array}{c}89.6 \\
(0.2587)\end{array}$ \\
\hline & & Bayes & $\begin{array}{c}93.4 \\
(0.2286)\end{array}$ & $\begin{array}{c}93.9 \\
(0.2598)\end{array}$ & $\begin{array}{c}94.2 \\
(0.2085)\end{array}$ & $\begin{array}{c}93.3 \\
(0.3108)\end{array}$ & $\begin{array}{c}93.8 \\
(0.1439)\end{array}$ & $\begin{array}{c}93.8 \\
(0.2314)\end{array}$ & $\begin{array}{c}93.3 \\
(0.1979)\end{array}$ & $\begin{array}{c}93.6 \\
(0.2436)\end{array}$ \\
\hline \multirow{6}{*}{$(50,20,25)$} & \multirow[b]{2}{*}{1} & MLE & $\begin{array}{c}90.5 \\
(0.2164)\end{array}$ & $\begin{array}{c}89.6 \\
(0.2561)\end{array}$ & $\begin{array}{c}89.6 \\
(0.2048)\end{array}$ & $\begin{array}{c}90.1 \\
(0.3099)\end{array}$ & $\begin{array}{c}90.3 \\
(0.1474)\end{array}$ & $\begin{array}{c}90.5 \\
(0.2186)\end{array}$ & $\begin{array}{c}91.4 \\
(0.1964)\end{array}$ & $\begin{array}{c}91.3 \\
(0.2486)\end{array}$ \\
\hline & & Bayes & $\begin{array}{c}95.4 \\
(0.2072)\end{array}$ & $\begin{array}{c}96.1 \\
(0.2337)\end{array}$ & $\begin{array}{c}95.6 \\
(0.1853)\end{array}$ & $\begin{array}{c}95.1 \\
(0.2876)\end{array}$ & $\begin{array}{c}96.3 \\
(0.1314)\end{array}$ & $\begin{array}{c}95.4 \\
(0.2089)\end{array}$ & $\begin{array}{c}95.1 \\
(0.1776)\end{array}$ & $95(0.2243)$ \\
\hline & \multirow[b]{2}{*}{2} & MLE & $\begin{array}{c}90.3 \\
(0.2195)\end{array}$ & $\begin{array}{c}89.5 \\
(0.2517)\end{array}$ & $\begin{array}{c}89.5 \\
(0.2015)\end{array}$ & $90(0.3093)$ & $\begin{array}{c}90.1 \\
(0.1481)\end{array}$ & $\begin{array}{c}90.1 \\
(0.2192)\end{array}$ & $\begin{array}{c}91.4 \\
(0.1947)\end{array}$ & $\begin{array}{c}91.2 \\
(0.2401)\end{array}$ \\
\hline & & Bayes & $\begin{array}{c}95.4 \\
(0.2095)\end{array}$ & $\begin{array}{c}95.8 \\
(0.2359)\end{array}$ & $\begin{array}{c}95.3 \\
(0.1877)\end{array}$ & $95(0.2892)$ & $\begin{array}{c}96.2 \\
(0.1287)\end{array}$ & $\begin{array}{c}95.3 \\
(0.2107)\end{array}$ & $\begin{array}{c}95.1 \\
(0.1795)\end{array}$ & $95(0.2261)$ \\
\hline & \multirow[b]{2}{*}{3} & MLE & $\begin{array}{c}90.2 \\
(0.2167)\end{array}$ & $\begin{array}{c}89.5 \\
(0.2538)\end{array}$ & $\begin{array}{c}89.4 \\
(0.2029)\end{array}$ & $\begin{array}{c}89.5 \\
(0.3085)\end{array}$ & $\begin{array}{c}89.7 \\
(0.1497)\end{array}$ & $\begin{array}{c}90.1 \\
(0.2213)\end{array}$ & $\begin{array}{c}91.3 \\
(0.1942)\end{array}$ & $\begin{array}{c}91.2 \\
(0.2389)\end{array}$ \\
\hline & & Bayes & $\begin{array}{c}94.3 \\
(0.2104)\end{array}$ & $\begin{array}{c}95.3 \\
(0.2413)\end{array}$ & $\begin{array}{c}95.2 \\
(0.1895)\end{array}$ & $95(0.2886)$ & $\begin{array}{c}96.1 \\
(0.1274)\end{array}$ & $\begin{array}{c}95.2 \\
(0.2171)\end{array}$ & $\begin{array}{c}95.1 \\
(0.1778)\end{array}$ & $\begin{array}{c}94.9 \\
(0.2216)\end{array}$ \\
\hline \multirow{6}{*}{$(50,25,30)$} & \multirow[b]{2}{*}{1} & MLE & $\begin{array}{c}92.7 \\
(0.1987)\end{array}$ & $\begin{array}{c}91.4 \\
(0.2356)\end{array}$ & $\begin{array}{c}91.3 \\
(0.1864)\end{array}$ & $\begin{array}{c}92.6 \\
(0.2885)\end{array}$ & $\begin{array}{c}91.4 \\
(0.1237)\end{array}$ & $\begin{array}{c}92.3 \\
(0.1993)\end{array}$ & $\begin{array}{c}92.3 \\
(0.1763)\end{array}$ & $93(0.224)$ \\
\hline & & Bayes & $\begin{array}{c}97.1 \\
(0.1857)\end{array}$ & $\begin{array}{c}97.3 \\
(0.2134)\end{array}$ & $\begin{array}{c}97.2 \\
(0.1646)\end{array}$ & $\begin{array}{c}97.2 \\
(0.2661)\end{array}$ & $\begin{array}{c}98.2 \\
(0.1114)\end{array}$ & $\begin{array}{c}97.5 \\
(0.1856)\end{array}$ & $\begin{array}{c}98.4 \\
(0.1575)\end{array}$ & $\begin{array}{c}97.8 \\
(0.2037)\end{array}$ \\
\hline & \multirow[b]{2}{*}{2} & MLE & $\begin{array}{c}92.6 \\
(0.1972)\end{array}$ & $\begin{array}{c}91.4 \\
(0.2364)\end{array}$ & $\begin{array}{c}91.1 \\
(0.1876)\end{array}$ & $\begin{array}{c}92.4 \\
(0.2869)\end{array}$ & $\begin{array}{c}91.4 \\
(0.1261)\end{array}$ & $\begin{array}{c}92.2 \\
(0.1927)\end{array}$ & $\begin{array}{c}92.2 \\
(0.1769)\end{array}$ & $\begin{array}{c}92.8 \\
(0.2222)\end{array}$ \\
\hline & & Bayes & $\begin{array}{c}97.1 \\
(0.1829)\end{array}$ & $\begin{array}{c}97.3 \\
(0.2155)\end{array}$ & $\begin{array}{c}97.2 \\
(0.1655)\end{array}$ & $\begin{array}{c}97.1 \\
(0.2658)\end{array}$ & $\begin{array}{c}98.2 \\
(0.1142)\end{array}$ & $\begin{array}{c}97.4 \\
(0.1887)\end{array}$ & $\begin{array}{c}98.3 \\
(0.1586)\end{array}$ & $\begin{array}{c}97.8 \\
(0.2062)\end{array}$ \\
\hline & \multirow[b]{2}{*}{3} & MLE & $\begin{array}{c}92.6 \\
(0.1957)\end{array}$ & $\begin{array}{c}91.4 \\
(0.2357)\end{array}$ & $\begin{array}{c}91.1 \\
(0.1835)\end{array}$ & $\begin{array}{c}92.4 \\
(0.2886)\end{array}$ & $\begin{array}{c}91.4 \\
(0.1274)\end{array}$ & $\begin{array}{c}92.2 \\
(0.2013)\end{array}$ & $\begin{array}{c}92.2 \\
(0.1733)\end{array}$ & $\begin{array}{c}92.8 \\
(0.2162)\end{array}$ \\
\hline & & Bayes & $\begin{array}{c}97.8 \\
(0.1848)\end{array}$ & $\begin{array}{c}97.3 \\
(0.2258)\end{array}$ & $\begin{array}{c}97.2 \\
(0.1642)\end{array}$ & $\begin{array}{c}97.1 \\
(0.2679)\end{array}$ & $\begin{array}{c}98.2 \\
(0.1145)\end{array}$ & $\begin{array}{c}97.4 \\
(0.1923)\end{array}$ & $\begin{array}{c}98.3 \\
(0.1566)\end{array}$ & $\begin{array}{c}97.8 \\
(0.2045)\end{array}$ \\
\hline
\end{tabular}

TABLE 6: The failure data with the dependent competing risks' model.

\begin{tabular}{lllllllll}
\hline$S_{1}$ & $(0.04,0)$ & $(0.10,1)$ & $(0.16,0)$ & $\left(0.17,{ }^{*}\right)$ & $(0.42,2)$ & $(0.46,0)$ & $\left(0.47,{ }^{*}\right)$ & $(0.47,1)$ \\
& $(1.05,1)$ & $(1.50,2)$ & $(1.80,0)$ & & & & \\
$S_{2}$ & $(0.02,1)$ & $(0.06,2)$ & $\left(0.27,{ }^{*}\right)$ & $(0.30,2)$ & $(0.30,2)$ & $(0.34,1)$ & $\left(0.36,,^{*}\right)$ & $(0.70,2)$ \\
& $(0.80,2)$ & $(0.80,0)$ & $\left(0.92,{ }^{*}\right)$ & $(1.06,2)$ & & & $(0.30,1)$ \\
$S_{3}$ & $(0.07,2)$ & $(0.13,1)$ & $\left(0.20,{ }^{*}\right)$ & $(0.29,1)$ & $(0.30,2)$ & $\left(0.30,{ }^{*}\right)$ & $(0.33,2)$ \\
\hline
\end{tabular}

TABLe 7: Estimations and 95\% confidence and incredible intervals.

\begin{tabular}{lcccc}
\hline Estimator & MLE & ACI & Bayes & HPD \\
\hline$\lambda_{1}$ & 0.3133 & $(0.0911,0.5982)$ & 0.3027 & $(0.0953,0.5976)$ \\
$\alpha_{10}$ & 1.7241 & $(0.9851,2.4724)$ & 1.7163 & $(0.9735,2.3853)$ \\
$\alpha_{11}$ & $(0.3953,1.1285)$ & 0.8473 & $(0.4075,1.1153)$ \\
$\alpha_{12}$ & 0.8616 & $(0.4261,1.1176)$ & 0.8193 & $(0.4378,1.1104)$ \\
$\lambda_{2}$ & 0.8247 & $(0.3956,1.1432)$ & 0.8287 & $(0.3918,1.1427)$ \\
$\alpha_{20}$ & $(0.6853,2.2589)$ & 1.4517 & $(0.6978,2.2667)$ \\
$\alpha_{21}$ & 0.8359 & $(0.4137,1.0134)$ & 0.7689 & $(0.4166,1.0089)$ \\
$\alpha_{22}$ & 1.4937 & $(0.6176,1.6792)$ & 1.0816 & $(0.6186,1.6811)$ \\
$\lambda_{3}$ & 0.7745 & $(0.5983,1.5925)$ & 1.0673 & $(0.6017,1.5937)$ \\
$\alpha_{30}$ & 1.1201 & $(1.4327,3.2896)$ & 2.2164 & $(1.4673,3.2576)$ \\
$\alpha_{31}$ & 1.1024 & $(0.8221,2.1074)$ & 1.4091 & $(0.8226,2.0974)$ \\
$\alpha_{32}$ & 2.3691 & $(0.8831,2.2146)$ & 1.4973 & $(0.8875,2.2121)$ \\
\hline
\end{tabular}


TABLE 8: The K-S statistics and the corresponding $p$ value.

\begin{tabular}{|c|c|c|c|c|c|c|c|c|c|}
\hline Distribution & $F\left(t ; \lambda_{1}, \alpha_{101}\right)$ & $F\left(t ; \lambda_{1}, \alpha_{102}\right)$ & $F\left(t ; \lambda_{1}, \alpha_{1}\right)$ & $F\left(t ; \lambda_{2}, \alpha_{201}\right)$ & $F\left(t ; \lambda_{2}, \alpha_{202}\right)$ & $F\left(t ; \lambda_{2}, \alpha_{2}\right)$ & $F\left(t ; \lambda_{3}, \alpha_{301}\right)$ & $F\left(t ; \lambda_{3}, \alpha_{302}\right)$ & $F\left(t ; \lambda_{3}, \alpha_{3}\right)$ \\
\hline $\mathrm{K}-\mathrm{S}$ & 0.2443 & 0.1435 & 0.1689 & 0.1515 & 0.1715 & 0.2035 & 0.1737 & 0.2089 & 0.2388 \\
\hline$p$ & 0.5562 & 0.9536 & 0.8620 & 0.9076 & 0.8157 & 0.6325 & 0.7676 & 0.6324 & 0.5873 \\
\hline
\end{tabular}

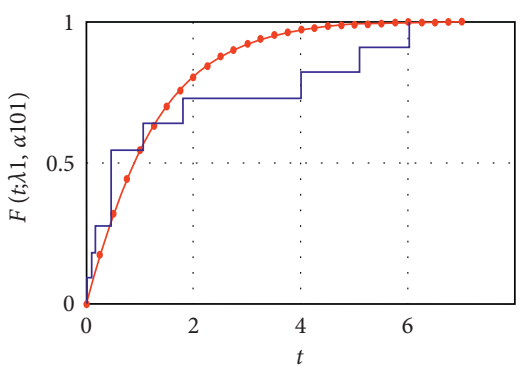

- K-S

$\rightarrow$ Gompertz

(a)

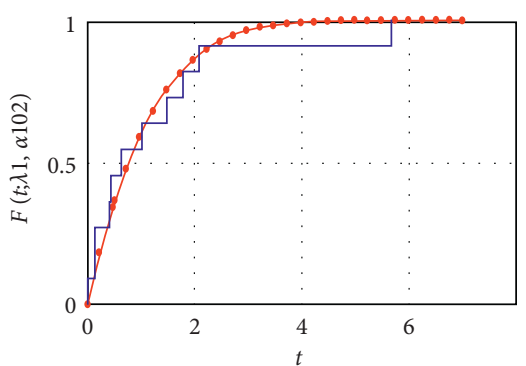

- K-S

$\rightarrow$ Gompertz

(b)

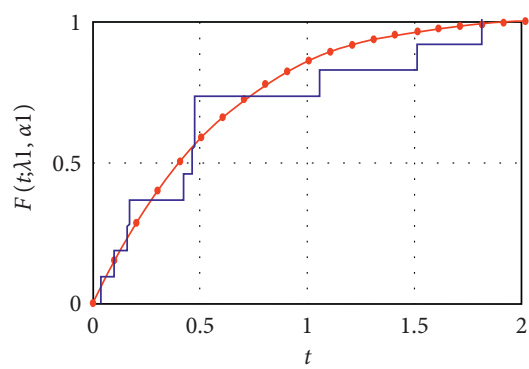

- K-S

$\multimap$ Gompertz

FIGURE 1: Comparison chart for empirical distribution and its fitted distribution under $S_{1}$ level. (a) The comparison chart when $\alpha=\alpha_{101}$.

(b) The comparison chart when $\alpha=\alpha_{102}$. (c) The comparison chart when $\alpha=\alpha_{1}$.

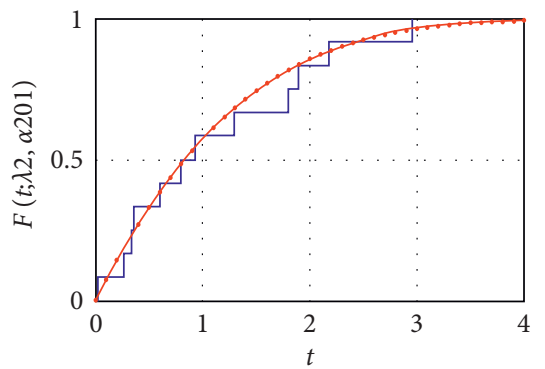

K-S

$\longrightarrow$ Gompertz

(a)

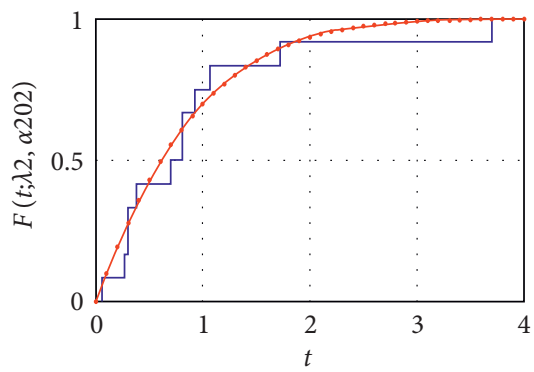

$-\mathrm{K}-\mathrm{S}$

$\longrightarrow$ Gompertz

(b)

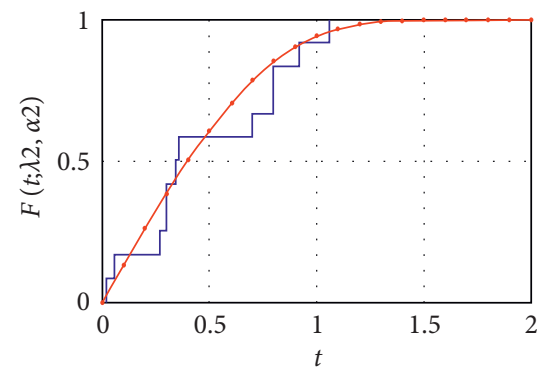

K-S

$\rightarrow$ Gompertz

(c)

FIGURE 2: Comparison chart for empirical distribution and its fitted distribution under $S_{2}$ level. (a) The comparison chart when $\alpha=\alpha_{201}$.

(b) The comparison chart when $\alpha=\alpha_{202}$. (c) The comparison chart when $\alpha=\alpha_{2}$.

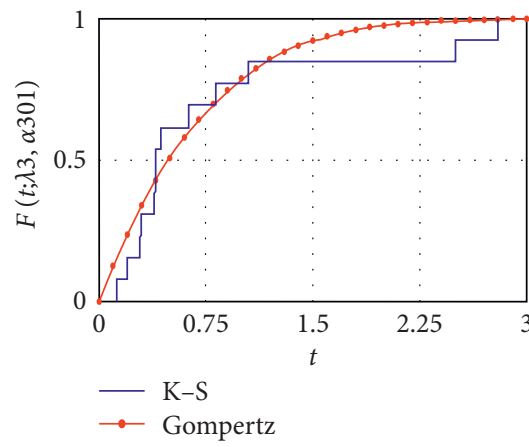

(a)

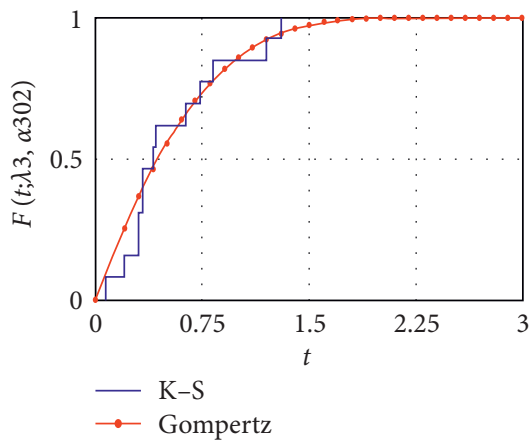

(b)

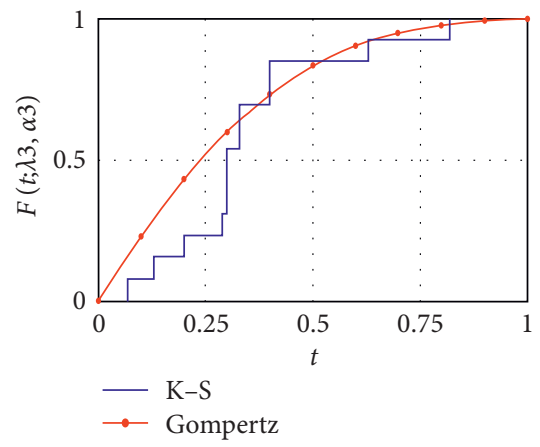

(c)

Figure 3: Comparison chart for empirical distribution and its fitted distribution under $S_{3}$ level. (a) The comparison chart when $\alpha=\alpha_{301}$.

(b) The comparison chart when $\alpha=\alpha_{302}$. (c) The comparison chart when $\alpha=\alpha_{3}$. 
From Table 8, we can obtain that all the corresponding $p$ values are bigger than the given significance value. It indicates that the failure data comes from the MOBG distribution.

\section{Conclusion}

Under the AT-II PHCS, we consider statistical analysis of the dependent competing risk model by using the MOBG distribution under CSALT in which the shape parameters of life distribution are changed with the stress levels. Moreover, we also consider the failure causes of some units are unknown in the dependent competing risks' model, and then, both the ML method and Bayes approach are used to obtain the estimations of the unknown parameters. Based on these works, the expected values and MSEs of MLEs and BEs are derived, and the AILs and CPs of the unknown parameters based on ACIs and HPD CIs are also obtained. Finally, the performances of all procedures are evaluated. The simulation results show that the MSEs, AIL, and CPs of the unknown parameters perform well, which are in the bigger sample size $n$ or effective sample size $m$. Furthermore, compared with the ML method, the Bayes approach is superior according to the MSEs, AIL, and CPs of the unknown parameters.

\section{Data Availability}

The data used to support the findings of this paper are included within the supplementary information file.

\section{Conflicts of Interest}

The authors declare that they have no conflicts of interest.

\section{Acknowledgments}

This work was supported by the National Natural Science Foundation of China under Grant nos. 71571144 and 71401134.

\section{References}

[1] P. C. Li, W. Ting, and D. L. Kwong, "Time-dependent dielectric breakdown of chemical-vapour-deposited SiO3gate dielectrics," Electronics Letters, vol. 25, no. 10, pp. 665-666, 1989.

[2] P. Hiergeist, A. Spitzer, and S. Rohl, "Lifetime of thin oxide and oxide-nitride-oxide dielectrics within trench capacitors for DRAMs," IEEE Transactions on Electron Devices, vol. 36, no. 5, pp. 913-919, 1989.

[3] G. Henrich and U. Jensen, "Parameter estimation for a bivariate lifetime distribution in reliability with multivariate extension,” Metrika, vol. 42, no. 1, pp. 49-65, 1995.

[4] Q. Guan, Y. Tang, and A. Xu, "Objective bayesian analysis for bivariate marshall-olkin exponential distribution," Computational Statistics \& Data Analysis, vol. 64, pp. 299-313, 2013.

[5] Y. Shen and $\mathrm{A}$. $\mathrm{Xu}$, "On the dependent competing risks using Marshall-Olkin bivariate Weibull model: parameter estimation with different methods," Communications in Statistics, vol. 47, pp. 5585-5572, 2018.
[6] X. Bai, Y. Shi, B. Liu, and Q. Fu, "Statistical inference of Marshall-Olkin bivariate Weibull distribution with three shocks based on progressive interval censored data," Communications in Statistics - Simulation and Computation, vol. 48, no. 3, pp. 637-654, 2019.

[7] D. Kundu and A. K. Gupta, "Bayes estimation for the Marshall-Olkin bivariate Weibull distribution," Computational Statistics \& Data Analysis, vol. 57, no. 1, pp. 271-281, 2013.

[8] S. H. Feizjavadian and R. Hashemi, "Analysis of dependent competing risks in the presence of progressive hybrid censoring using Marshall-Olkin bivariate Weibull distribution," Computational Statistics \& Data Analysis, vol. 82, pp. 19-34, 2015.

[9] G. E. Dinse, "Nonparametric estimation for partially-complete time and type of failure data," Biometrics, vol. 38, no. 2, pp. $417-431,1982$.

[10] M. Miyakawa, "Statistical analysis of incomplete data in competing risks model," The Japanese Society for Quality Control, vol. 33, pp. 293-296, 1982.

[11] H. K. T. Ng, D. Kundu, and P. S. Chan, "Statistical analysis of exponential lifetimes under an adaptive Type-II progressive censoring scheme," Naval Research Logistics (NRL), vol. 56, no. 8, pp. 687-698, 2009.

[12] E. Cramer and G. Iliopoulos, "Adaptive progressive Type-II censoring," Test, vol. 19, no. 2, pp. 342-358, 2010.

[13] F. Hemmati and E. Khorram, "On adaptive progressively type-II censored competing risks data," Communications in Statistics - Simulation and Computation, vol. 46, no. 6, pp. 4671-4693, 2017.

[14] M. Nassar and O. E. Abo-Kasem, "Estimation of the inverse Weibull parameters under adaptive type-II progressive hybrid censoring scheme," Journal of Computational and Applied Mathematics, vol. 315, pp. 228-239, 2017.

[15] B. Gompertz, "On the nature of the function expressive of the law of human mortality and onthe new mode of determining the value of life contingencies," Philosophical Transactions of the Royal Society A, vol. 115, pp. 513-580, 1825.

[16] G. Doblhammer, "Reproductive history and mortality later in life: a comparative study of England and wales and Austria," Population Studies, vol. 54, no. 2, pp. 169-176, 2000.

[17] M. Perozek, "Using subjective expectations to forecast longevity: do survey respondents know something we don't know?," Demography, vol. 45, no. 1, pp. 95-113, 2008.

[18] W. J. Willemse and H. Koppelaar, "Knowledge elicitation of Gompertz' law of mortality," Scandinavian Actuarial Journal, vol. 2000, no. 2, pp. 168-179, 2000.

[19] C. E. Finch and M. C. Pike, "Maximum life span predictions from the Gompertz mortality model," The Journals of Gerontology Series A: Biological Sciences and Medical Sciences, vol. 51, no. 3, p. B183, 1996.

[20] R. E. Ricklefs and A. Scheuerlein, "Biological implications of the Weibull and Gompertz models of aging," The Journals of Gerontology Series A: Biological Sciences and Medical Sciences, vol. 57, no. 2, pp. B69-B76, 2002.

[21] M. Wu and Y. Shi, "Bayes estimation and expected termination time for the competing risks model from Gompertz distribution under progressively hybrid censoring with binomial removals," Journal of Computational and Applied Mathematics, vol. 300, pp. 420-431, 2016.

[22] Y. Liu, Y. Shi, X. Bai, and B. Liu, "Stress-strength reliability analysis of system with multiple types of components using survival signature," Journal of Computational and Applied Mathematics, vol. 342, pp. 375-398, 2018. 
[23] M. M. Mohie El-Din, M. Nagy, and M. H. Abu-Moussa, "Estimation and prediction for Gompertz distribution under the generalized progressive hybrid censored data," Annals of Data Science, vol. 6, no. 4, p. 673, 2019.

[24] S. Dey, F. A. Moala, and D. Kumar, "Statistical properties and different methods of estimation of Gompertz distribution with application," Journal of Statistics and Management Systems, vol. 21, no. 5, pp. 839-876, 2018.

[25] N. Ebrahimi, "Analysis of bivariate accelerated life test data for the bivariate exponential distribution," American Journal of Mathematical and Management Sciences, vol. 7, no. 1-2, pp. 175-190, 1987.

[26] P. Congdon, Bayesian Statistical Modeling, John Wiley \& Sons, Hoboken, NJ, USA, 2nd Edition, 2010.

[27] D. Kundu and A. K. Gupta, "On bivariate Weibull-geometric distribution," Journal of Multivariate Analysis, vol. 123, no. 1, pp. 19-29, 2014. 